\title{
Optimal Design for Problems Involving Flow and Transport Phenomena in Saturated Subsurface Systems
}

\author{
Alex S. Mayer ${ }^{1}$ \\ Department of Geological Engineering and Sciences, Michigan Technological \\ University, Houghton, Michigan 49931-1295, USA \\ C.T. Kelley \\ Center for Research in Scientific Computation, Department of Mathematics, \\ North Carolina State University, Raleigh, North Carolina 27695-8205, USA
}

Cass T. Miller

Center for the Advanced Study of the Environment, Department of Environmental Sciences and Engineering, University of North Carolina, Chapel Hill, North Carolina 27599-7431, USA

\begin{abstract}
Estimation problems arise routinely in subsurface hydrology for applications that range from water resources management to water quality protection to subsurface restoration. Interest in optimal design of such systems has increased over the last two decades and this area is considered an important and active area of research. In this work, we review the state of the art, assess important challenges that must be resolved to reach a mature level of understanding, and summarize some promising approaches that might help meet some of the challenges. While much has been accomplished to date, we conclude that more work remains before comprehensive, efficient, and robust solution methods exist to solve the most challenging applications in subsurface science. We suggest that future directions of research include the application of direct search solution methods, and developments in stochastic and multi-objective optimization. We present a set of comprehensive test problems for use in the research community as a means for benchmarking and comparing optimization approaches.
\end{abstract}

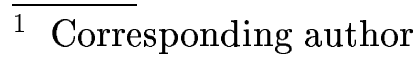

2 E-mail addresses: asmayer@mtu.edu (A.S. Mayer), Tim_Kelley@ncsu.edu (C.T. Kelley), casey_miller@unc.edu (C. T. Miller)

Preprint submitted to Elsevier Preprint $\quad 30$ April 2002 


\section{Notation}

\section{Roman Letters}

\begin{tabular}{|c|c|}
\hline$a_{k}$ & weighting coefficients in multiobjective formulation \\
\hline$b_{j}$ & exponential coefficients in cost objective functions \\
\hline$c_{j}$ & coefficients in cost objective functions \\
\hline$C_{0}^{\iota}$ & initial contaminant distribution for species $\iota$ \\
\hline$C_{s}^{\iota}$ & source or steady state concentration for species $\iota$ \\
\hline$C^{s w}$ & concentration of solute in seawater \\
\hline$C_{b}^{\iota}$ & background concentration of solute in freshwater \\
\hline$C_{i}^{\iota}$ & concentration in extraction well $i$ for species $\iota$ \\
\hline$C_{E}^{\iota}$ & target effluent concentration from treatment process for species $\iota$ \\
\hline $\mathbf{D}$ & hydrodynamic dispersion tensor \\
\hline$D^{\iota *}$ & free liquid diffusivity of species $\iota$ \\
\hline$d_{i}$ & depth of well below the ground surface \\
\hline$f, f_{k}$ & objective function \\
\hline$f^{c}$ & capital costs \\
\hline$f^{o}$ & operational costs \\
\hline$f^{R}$ & measure of reliability \\
\hline$\tilde{f}$ clean & pdf for cleanup performance \\
\hline$\tilde{f}^{\cos t}$ & pdf for cost function \\
\hline$F$ & multi-objective functional \\
\hline$F_{s w}$ & allowable solute concentration normalized by seawater concentration \\
\hline g & mathematical model \\
\hline $\mathbf{g}_{0}$ & initial conditions for $\mathbf{g}$ \\
\hline $\mathbf{g}_{b}$ & boundary conditions for $\mathbf{g}$ \\
\hline$g$ & magnitude of gravitational acceleration, oriented in the $-\mathbf{k}$ direction \\
\hline$h$ & hydraulic head, \\
\hline$h^{\max }$ & maximum allowable head \\
\hline$h^{\min }$ & minimum allowable head \\
\hline$h_{i}$ & head in well $i$ \\
\hline$h_{s}$ & steady-state solution to the unperturbed flow problem \\
\hline $\mathcal{I}$ & interphase mass exchange \\
\hline $\mathcal{I}^{\iota}$ & interphase mass transfer relations \\
\hline $\mathbf{k}$ & effective permeability tensor \\
\hline $\mathbf{K}$ & hydraulic conductivity tensor \\
\hline$K$ & hydraulic conductivity scalar \\
\hline$k_{f}$ & first-order mass transfer rate coefficient \\
\hline$K_{f}$ & Freundlich capacity coefficient \\
\hline$M$ & maximum fractional mass remaining after remediation \\
\hline$n$ & number of wells \\
\hline$n^{e}$ & number of extraction wells \\
\hline$n_{f}$ & exponent related to the sorption energy \\
\hline
\end{tabular}




\begin{tabular}{ll}
$n_{k}$ & number of objective functions \\
$n^{i}$ & number of injection wells \\
$\mathbf{p}$ & set of model parameters \\
$\mathbf{q}$ & Darcy velocity, or volumetric flux \\
$p$ & fluid pressure \\
$q_{y}, q_{z}$ & Darcy flux out of the domain \\
$Q_{i}^{m}$ & design extraction rate at well $i$ \\
$Q^{e m a x}$ & maximum extraction rate \\
$Q^{i m a x}$ & maximum injection rate \\
$Q_{T}$ & total net pumping rate rate \\
$Q_{T}^{e}$ & total extraction rate \\
$Q_{T}^{\text {max }}$ & maximum total extraction rate \\
$Q_{T}^{\text {min }}$ & minimum total extraction rate \\
$Q_{i}$ & extraction or injection rate for well $i$ \\
$r$ & $Q_{i}<0$ indicates extraction and $Q_{i}>0$ indicates injection \\
$\mathcal{R}$ & species qualifier for the sorbing species \\
$S_{s}$ & biological or chemical reactions \\
$\mathcal{S}$ & specific storage coefficient \\
$\mathcal{S}$ & solute source term \\
$t$ & fluid source term \\
$t_{f}$ & time \\
$t_{s}$ & operation or remediation time \\
$\mathbf{u}$ & plume development time \\
$\mathbf{v}$ & vector of state variables \\
$\mathbf{w}$ & mean pore fluid flow velocity vector \\
$\mathbf{x}$ & vector of decision variables \\
$\mathbf{x}_{\mathbf{s}}$ & position vector \\
$\mathbf{x}_{\mathbf{i}}$ & location of contaminant source \\
$\mathbf{z}$ & well locations \\
$z$ & vector of decision variables \\
$z_{g s}$ & spatial coordinate oriented aligned with $\mathbf{k}$ \\
$Z$ & elevation of ground surface \\
& height of air stripping tower \\
& \\
\hline &
\end{tabular}

Greek Letters

$\begin{array}{ll}\alpha_{l} & \text { longitudinal dispersivity } \\ \alpha_{t} & \text { transverse dispersivity } \\ \eta_{x}, \eta_{y}, \eta_{z} & \text { spatial correlation scales } \\ \mu & \text { dynamic viscosity } \\ \mu_{\log K} & \text { mean } K \text { in } \log _{10} \text { space } \\ \nu, \nu_{x}, \nu_{y}, \nu_{z} & \text { spatial coordinates in covariance model } \\ \omega & \text { mass fraction } \\ \omega^{r s e} & \text { equilibrium solid-phase mass fraction }\end{array}$




$\begin{array}{ll}\Omega_{u} & \text { model domain } \\ \Omega_{u} & \text { feasible region of } \mathbf{u} \\ \Omega_{w} & \text { feasible region of } \mathbf{w} \\ \Omega_{z} & \text { feasible region of } \mathbf{z} \\ \rho & \text { density } \\ \rho_{0} & \text { freshwater density } \\ \rho_{s w} & \text { seawater density } \\ \sigma_{\log K}^{2} & \text { variance of } K \text { in } \log _{10} \text { space. } \\ \tau & \text { tortuosity of the porous medium } \\ \theta & \text { volume fraction }\end{array}$

Subscripts

$i \quad$ well index

$k \quad$ objective function index

$s \quad$ qualifier denoting source or steady state

$\alpha \quad$ phase index

Superscripts

a qualifier denoting aqueous phase

$e \quad$ qualifier denoting extraction

$i \quad$ qualifier denoting injection

$s \quad$ qualifier denoting solid phase

$\iota \quad$ species index

\section{Abbreviations}

$\mathrm{BIO} \quad$ engineered bioremediation

CNSMOP constrained, nonlinear, stochastic, multi-objective optimization problems

COM combinatorial method

D $\quad \Omega_{z}$ includes decision variable

DS direct search

DYL dynamic, linear $f$ form

DYLP dynamic, linear programming

DYNL dynamic, nonlinear $f$ form

DYNLP dynamic, nonlinear programming

EA evolutionary algorithm

GAC granular activated carbon

GLO global approximation

$\mathrm{HC} \quad$ hydraulic capture 
$f$ form includes integer term

L $\quad$ linear $f$ form

LF $\quad g$ form includes linear flow

LP linear programming

LS $\quad \Omega_{z}$ includes state variable, linear

LT $\quad g$ form includes linear transport

MO $\quad f$ form considers multiple objectives

MS $\quad g$ form includes multiple species

NL nonlinear $f$ form

NLF

NLP

$\mathrm{g}$ form includes nonlinear flow

NLS nonlinear programming

NLT

PAT

$\Omega_{z}$ includes state variable, nonlinear

PDF $\mathrm{g}$ form includes nonlinear transport

RS pump and treat

RX

STO

SVE

WS probability distribution function response surface used as solution of $\mathbf{g}$ form $\mathrm{g}$ form includes non-conservative transport stochastic variable(s) in $\mathbf{g}$ form soil vapor extraction water supply 


\section{INTRODUCTION}

Modeling of fluid flow and contaminant transport in subsurface porous medium systems has become commonplace [128]. These simulations continue to advance our general understanding of transport phenomena and are increasingly used as a basis for managing subsurface systems. Such management may involve identifying model parameters [84, 132], planning data collection programs [14, 80], or designing and operating engineered systems [66]. Within this general class of problems, design problems associated with fluid flow and species transport in subsurface systems are especially important [102].

Broadly speaking, design problems for these applications fall into the general class of constrained, nonlinear, stochastic, multi-objective optimization problems (CNSMOP) — an especially challenging class of application. The stochastic nature of these problems results directly from our imperfect knowledge of model parameters, auxiliary conditions, or in some cases even the fundamental form of the model that governs transport phenomena in typical heterogeneous porous medium systems [101]. The multi-objective aspect of these problems arises, for example, from a conflicting desire to minimize cost and maximize reliability of a design [116].

Most efforts to date for subsurface optimization problems have been some simple subset of the general class of CNSMOP problems. This is largely because of the complexity and computational demands of solving this general class of problems and partly because of the evolving nature of stochastic optimization. Over the last two decades, groundwater quality control and remediation have been the focus of optimization efforts in the subsurface literature $[4,5,7,6,87,43,82,93,134,66,80,118,8,3,133,92,90,147$, $74,113,83,110,45,92,102,13,106,12,79]$; in particular, the design of pump-and-treat (PAT) systems is the most frequent application considered $[4,5,7,6,87,93,74,118,3,83,10,79,23,92,12,13,90,121]$.

The objectives of this work are (1) to provide a common conceptual framework for subsurface design problems; (2) to consider the state of knowledge in the light of this framework; (3) to highlight limitations of existing approaches used to solve subsurface design problems; (4) to introduce evolving approaches from other fields that may be of use in advancing our study of subsurface design problems; and (5) to introduce a set of problems that could further the development and testing of methods for solving subsurface design problems. 


\section{PROBLEM STATEMENT}

The problem of finding optimal designs for subsurface systems can be formulated as

$$
\min _{\mathbf{z} \in \Omega_{z}} f(\mathbf{z})
$$

where the objective function $f$ is assumed with a minimization convention, $\mathbf{z}(\mathbf{u}, \mathbf{w})$ is a vector of decision variables consisting of a vector $\mathbf{u}$ of state variables and a vector $\mathbf{w}$ of decision variables, $\Omega_{z}=\Omega_{u} \cup \Omega_{w}$ is the feasible region of $\mathbf{z}$ represented by a set of constraint equations, $\Omega_{u}$ represents the feasible region of $\mathbf{u}$, and $\Omega_{w}$ represents the feasible region of $\mathbf{w}$. The constraints, $\Omega_{u}$ and $\Omega_{w}$, are specified for state and decision variables as a way to enforce practical, technological constraints or to reduce the size or complexity of the optimization problem. The objective function $f$ can include one or more than one function $f_{k}$, where $k \leq K$ represents the number of discrete objective functions. When $K>1$, the problem is termed a multi-objective (MO) problem. An MO problem would arise when separate objective functions are considered that are conflicting and not convertible to the same units, i.e. cost (\$) vs. risk (increased incidence of disease).

The decision variables for subsurface flow and transport applications can include both real number quantities, integers, and categorical variables related to the design problem of interest. For example, real number decision variables might be associated with injection or withdrawal flow rates, the mass rate of injection of a species added to encourage a desired reaction, and variables describing the size and location of remediation unit processes. Integer variables could include the number of injection or withdrawal wells. Categorical variables are discrete quantities that are a part of the design space and for which only certain values may be specified in order for the objective function to be evaluated and return a valid quantity. An example of a categorical variable would be a type of design or a unit process from a set of possible processes.

State variables represent the state of the physical system being considered, which could include fluid pressures or species concentrations. For the problems of concern, the state of the physical system is typically represented by a mechanistic mathematical model consisting of a set of conservation equations and closure relations, which take the form of a set of differential equations and auxiliary conditions. We represent our mathematical model as

$$
\mathbf{g}=\mathbf{g}\left(\mathbf{x}, t ; \mathbf{g}_{0}, \mathbf{g}_{b}, \mathbf{p}\right)
$$


where $\mathbf{x}$ is a position vector, $t$ is time, $\mathbf{g}_{0}$ represents initial conditions, $\mathbf{g}_{b}$ represents the boundary conditions, and $\mathbf{p}$ represents a set of model parameters, which may include decision variables $\mathbf{w}$, thus $\mathbf{w} \subset\left(\mathbf{p} \cup \mathbf{g}_{b}\right)$. The mathematical model may also include components not represented by a mechanistic model; for example, an empirical model may be used to represent a treatment process.

While the standard approach is for $\mathbf{g}$ to represent a deterministic model of a physical system, such representations of natural systems are often unreliable. This is so because $\mathbf{g}_{0}, \mathbf{g}_{b}$, and $\mathbf{p}$ are not known with certainty. Under such conditions, $\mathbf{g}$ is best represented by a stochastic mathematical model, leading to a stochastic $\mathbf{u}$, and hence a stochastic $f$ function. Such so-called stochastic optimization problems are difficult to solve. In the simplest stochastic conceptualization, we can imagine $\mathbf{u}$ as a probability density function (PDF, $P_{u}$ ) and $f$ as being linearly dependent on $\mathbf{u}$. In this case, we can determine a PDF for $f$ that is simply a linear transformation of $P_{u}$. However, the real situation is considerably more complex - $\mathbf{u}$ may not be described with an analytical $\mathrm{PDF}, f$ is unlikely to depend on $\mathbf{u}$ in a linear fashion, and $\Omega_{z}$ may need to be specified in a stochastic manner as well. In addition to the mathematical and algorithmic complexities resulting from a stochastic $\mathbf{g}$, it may be costly to compute the solution to a stochastic mathematical model $\mathbf{g}$.

The objective function $f$ is a functional that depends upon $\mathbf{g}$ and perhaps other functions. Since the mathematical form of $f$ is important in choosing the appropriate optimization solution method, the properties of all components

of $f$, including $\mathbf{g}$, and $\Omega_{z}$ are important. It is tempting to apply simplifying assumptions such that $f$ is deterministic, convex, and linear. It is our opinion that such simplified representations might be useful for a first-cut analysis, but that real problems are typically nonlinear and most appropriately represened using a stochastic approach - suggesting that solution methods for solving such noisy optimization problems are needed.

\section{RECENT APPROACHES AND REMAINING CHALLENGES}

Significant work has been performed over the last two decades on optimal design problems associated with subsurface flow and transport phenomena. These works can be classified in a number of ways: the technological application; the mathematical representation of the physical, chemical and biological systems, $\mathbf{g}$; the form of the objective function to be minimized, $f$; the form of the constraints, $\Omega_{z}$; and the methods used to solve the optimization problem. Table 1 is a summary of subsurface design problems organized according to this framework, first by application and second by model form. All abbreviations used in this table are defined in the notation section. We rely upon this tabulation to discuss the work accomplished to date and highlight unresolved 
problems. The works cited in this table and throughout the text are a comprehensive and representative, but not an exhaustive, survey of the literature.

Table 1: Summary of Subsurface Design Approaches

\begin{tabular}{|c|c|c|c|c|c|}
\hline Application & $\mathrm{g}$ form & $f$ form & $\Omega_{z}$ form & $\begin{array}{l}\text { Solution } \\
\text { Approach }\end{array}$ & Reference \\
\hline water supply & NLF, LT & $\mathrm{MO}, \mathrm{L}$ & $\mathrm{D}, \mathrm{NLS}$ & NLP, RS & {$[66]$} \\
\hline water supply & STO, LF & DYL & D, NLS & RS & [14] \\
\hline water supply & $\mathrm{LF}, \mathrm{LT}$ & NL & $\mathrm{D}, \mathrm{NLS}$ & NLP & {$[113]$} \\
\hline hydraulic capture & LF & $\mathrm{L}$ & $\mathrm{D}, \mathrm{LS}$ & LP & {$[8]$} \\
\hline hydraulic capture & LF & $\begin{array}{l}\text { MO, } \\
\text { L/NL }\end{array}$ & $\mathrm{D}$ & LP & {$[56]$} \\
\hline hydraulic capture & NLF & $\mathrm{L}$ & LS & NLP & {$[134]$} \\
\hline hydraulic capture & NLF & $\mathrm{L}$ & $\mathrm{D}, \mathrm{NLS}$ & NLP & {$[106]$} \\
\hline hydraulic capture & $\mathrm{LF}, \mathrm{LT}$ & $\mathrm{I}, \mathrm{L}$ & $\mathrm{D}, \mathrm{LS}$ & $\mathrm{COM}$ & {$[122]$} \\
\hline hydraulic capture & $\begin{array}{l}\text { STO, LF, } \\
\text { LT }\end{array}$ & NL & $\mathrm{D}, \mathrm{LS}$ & NLP & {$[139]$} \\
\hline hydraulic capture & $\begin{array}{l}\text { STO, LF, } \\
\text { NLT }\end{array}$ & NL & $\mathrm{D}$ & NLP & {$[146]$} \\
\hline hydraulic capture & STO, LF & $\mathrm{I}, \mathrm{L}$ & $\mathrm{L}$ & LP/EA & {$[147]$} \\
\hline hydraulic capture & STO, LF & $\mathrm{MO}, \mathrm{L}$ & $\mathrm{D}$ & LP & {$[94]$} \\
\hline hydraulic capture & STO, NLF & $\mathrm{L}$ & $\mathrm{D}, \mathrm{LS}$ & NLP & {$[105]$} \\
\hline pump \& treat & $\mathrm{LF}, \mathrm{LT}$ & $\mathrm{L}$ & $\mathrm{D}, \mathrm{NLS}$ & NLP & {$[10]$} \\
\hline pump \& treat & $\mathrm{LF}, \mathrm{LT}$ & $\mathrm{L}$ & $\mathrm{D}, \mathrm{NLS}$ & NLP & {$[5]$} \\
\hline pump \& treat & $\mathrm{LF}, \mathrm{LT}$ & $\mathrm{L}$ & $\mathrm{D}, \mathrm{NLS}$ & NLP & {$[4]$} \\
\hline pump \& treat & LF, LT & $\mathrm{L} / \mathrm{NL}$ & $\mathrm{D}, \mathrm{NLS}$ & NLP & {$[7]$} \\
\hline pump \& treat & $\mathrm{LF}, \mathrm{LT}$ & $\mathrm{L} / \mathrm{NL}$ & $\mathrm{D}, \mathrm{NLS}$ & NLP & {$[6]$} \\
\hline pump \& treat & $\mathrm{LF}, \mathrm{LT}$ & DYNL & $\mathrm{D}, \mathrm{NLS}$ & DYNLP & {$[92]$} \\
\hline pump \& treat & $\mathrm{LF}, \mathrm{LT}$ & $\begin{array}{l}\text { I, } \\
\text { DYNL }\end{array}$ & $\mathrm{D}, \mathrm{NLS}$ & EA & {$[79]$} \\
\hline pump \& treat & $\mathrm{LF}, \mathrm{LT}$ & I, NL & $\mathrm{D}, \mathrm{NLS}$ & NLP & {$[140]$} \\
\hline
\end{tabular}

continued on next page 
Table 1: continued

\begin{tabular}{|l|l|l|l|l|l|}
\hline Application & g form & $f$ form & $\Omega_{z}$ form & $\begin{array}{l}\text { Solution } \\
\text { Approach }\end{array}$ & References \\
\hline \hline pump \& treat & LF, LT & I, NL & D, NLS & $\begin{array}{l}\text { NLP/EA, } \\
\text { RS }\end{array}$ & {$[12]$} \\
\hline pump \& treat & LF, LT & I, NL & D, NLS & GLO & {$[83]$} \\
\hline pump \& treat & LF, LT & $\begin{array}{l}\text { MO, I, } \\
\text { NL }\end{array}$ & D, NLS & EA & {$[51]$} \\
\hline pump \& treat & LF, LT & MO, L & D, LS & NLP & {$[3]$} \\
\hline pump \& treat & LF, LT & NA & D, NLS & NA & {$[9]$} \\
\hline pump \& treat & NLF, LT & DYNL & D, NLS & GLO & {$[117]$} \\
\hline pump \& treat & NLF, LT & I, NL & D, NLS & GLO & {$[82]$} \\
\hline pump \& treat & NLF, LT & MO, I, L & D, NLS & EA & {$[93]$} \\
\hline pump \& treat & NLF, LT & DYNL & D, NLS & DYNLP & {$[90]$} \\
\hline pump \& treat & $\begin{array}{l}\text { LF, NLT, } \\
\text { MS, RX }\end{array}$ & L & NLS & NLP & {$[74]$} \\
\hline pump \& treat & LF, NLT & DYL & D, LS & DYNLP & {$[43]$} \\
\hline pump \& treat & NLF, NLT & I, NL & D, NLS & NLP & {$[98]$} \\
\hline pump \& treat & $\begin{array}{l}\text { STO, LF, } \\
\text { LT }\end{array}$ & MO, I, & DY, NLS & NLP & {$[23]$} \\
\hline pump \& treat & $\begin{array}{l}\text { STO, LF, } \\
\text { LT }\end{array}$ & $\begin{array}{l}\text { MO, I, } \\
\text { NL }\end{array}$ & D, NLS & EA, RS & {$[13]$} \\
\hline pump \& treat & $\begin{array}{l}\text { STO, LF, } \\
\text { LT }\end{array}$ & I, NL & D, NLS & NLP & {$[123]$} \\
\hline pump \& treat & $\begin{array}{l}\text { STO, LF, } \\
\text { LT }\end{array}$ & I, NL & D, NLS & EA & {$[95]$} \\
\hline pump \& treat & $\begin{array}{l}\text { STO, LF, } \\
\text { LT }\end{array}$ & L & D, NLS & NLP & {$[138]$} \\
\hline pump \& treat & $\begin{array}{l}\text { STO, NLF, } \\
\text { LT }\end{array}$ & DYNL & D, NLS & DYNLP & {$[87]$} \\
\hline
\end{tabular}

continued on next page 
Table 1: continued

\begin{tabular}{|c|c|c|c|c|c|}
\hline Application & $\mathrm{g}$ form & $f$ form & $\Omega_{z}$ form & $\begin{array}{l}\text { Solution } \\
\text { Approach }\end{array}$ & References \\
\hline pump \& treat & $\begin{array}{l}\text { STO, NLF, } \\
\text { LT }\end{array}$ & NL & $\mathrm{D}, \mathrm{NLS}$ & $\mathrm{COM}$ & {$[121]$} \\
\hline bioremediation & $\begin{array}{l}\text { LF, NLT, } \\
\text { MS, RX }\end{array}$ & DYNL & D, NLS & DYNLP & {$[102]$} \\
\hline bioremediation & $\begin{array}{l}\text { LF, NLT, } \\
\text { MS, RX }\end{array}$ & DYNL & D, NLS & DYNLP/EA & {$[144]$} \\
\hline bioremediation & $\begin{array}{ll}\text { STO, LF, } \\
\text { NLT, MS, } \\
\text { RX }\end{array}$ & DYNL & D, NLS & $\mathrm{EA}, \mathrm{RS}$ & {$[76]$} \\
\hline bioremediation & $\begin{array}{ll}\text { STO, LF, } \\
\text { NLT, MS, } \\
\text { RX }\end{array}$ & DYNL & $\mathrm{D}, \mathrm{NLS}$ & DYNLP & {$[103]$} \\
\hline soil vapor ext. & $\begin{array}{l}\text { NLF, NLT, } \\
\text { RX, MS }\end{array}$ & $\mathrm{I}, \mathrm{NL}$ & $\mathrm{D}, \mathrm{NLS}$ & $\mathrm{COM}$ & {$[133]$} \\
\hline soil vapor ext. & NLF & DYNL & $\mathrm{D}, \mathrm{NLS}$ & $\begin{array}{l}\text { DYNLP, } \\
\text { EA, }\end{array}$ & {$[75]$} \\
\hline
\end{tabular}

\subsection{Applications}

In Table 1, we have focused primarily on subsurface remediation applications. The applications listed in Table 1 indicate that the most frequent application of optimization in subsurface flow and transport problems are hydraulic control (HC) and PAT. The goal of HC is to control the spread of a contaminant via manipulation of groundwater levels and flow directions. In PAT remediation, the goal is to reduce the contaminant mass in the subsurface, in order to meet a target concentration or global mass fraction. As in most subsurface optimization problems, including water supply optimization, the decision variables for HC and PAT consist of the number of wells $n$ and the corresponding extraction or rates $Q_{i}$ and locations, $\mathbf{x}_{i}$, or

$$
\mathbf{w}=\left(n, Q_{i}, \mathbf{x}_{i}\right) \text { for } i=1, \ldots n
$$

Each of the decision variables $\left(n, Q_{i}, \mathbf{x}_{i}\right)$ can change with time. In Table 1 , time-varying, or dynamic, approaches are indicated by DYL and DYLP $f$ 
forms and DYNL and DYNLP solution methods. Dynamic pumping rates have been shown to result in less expensive PAT designs compared to fixed pumping rate designs [e.g. 70, 31]. Dynamic decision variables also allow the flexibility to handle design goals that change with time; for example, the initial goal may be to capture the contaminant plume, followed later by the goal of reducing the contaminant mass [145].

Table 1 indicates that remediation technologies other than $\mathrm{HC}$ and PAT have been considered in the subsurface remediation literature. In situ bioremediation (BIO) is an established subsurface remediation technology strategy that is effective for certain, readily degradable classes of compounds. In situ bioremediation, like PAT, involves extracting and injecting water to control the flow system. Unlike PAT, the injection also supplies certain chemical species, such as electron acceptors and nutrients, that are needed for the biochemical reaction to occur and which are expected to be rate limiting for the application at hand. The computational effort required to optimize a bioremediation design is significantly greater than that for a PAT design, because additional transport equations need to be solved and additional decision variables must be considered [102]. Several strategies have been attempted to overcome these difficulties, such as assuming that oxygen is the single rate-limiting injectant and ignoring the additional decision variables [102].

Soil vapor extraction (SVE) is frequently used to remove volatile contaminants from regions above the water table. SVE involves pumping of air through contaminated media, in order to strip volatile contaminants from trapped liquid phases. The decision variables for SVE are similar to those for the PAT problem - well locations, extraction rates, and number of wells. Investigations of SVE optimization have focused on formulating the problem such that the optimization is robust and converges quickly [133, 75]. Other technologies where mathematical optimization has been applied include surfactant flushing [89], and combining thermal oxidation with PAT [119].

To date, each subsurface remediation technology (HC, PAT, BIO, SVE, etc.) has been treated separately when mathematical optimization has been applied to technology design. Furthermore, there are many more subsurface remediation technologies where optimization has not yet been applied in the design process. These limitations may in turn limit the widespread applicability of mathematical optimization to remediation design, since the design process includes choosing the appropriate remediation technology or combination of technologies. A separate line of research has been dedicated in recent decades to develop tools for choosing among remediation technologies [e.g. 1]). These tools typically take the form of user-friendly software packages, but have limited design capabilities. 


\subsection{Mathematical Model}

The mathematical model $\mathbf{g}$ for the problems of concern represents fluid flow and, in some cases, species transport and reaction problems in the subsurface, along with any ex situ processes (e.g. water treatment) that are a part of the system to be designed. The subsurface flow and transport processes can be described by a wide range of mechanistic models, which can abstractly be represented by a set of conservation equations and general closure relations, which can assume a wide range of specific forms.

Standard mathematical models for isothermal systems include some set of mass conservation equations and approximate momentum conservation equations of a form given by Darcy's law [101]. The conservation of mass for a species $\iota$ in a phase $\alpha$ is

$$
\frac{\partial}{\partial t}\left(\theta^{\alpha} \rho^{\alpha} \omega^{\iota \alpha}\right)=-\nabla \cdot\left(\theta^{\alpha} \rho^{\alpha} \omega^{\iota \alpha} \mathbf{v}^{\alpha}\right)-\nabla \cdot \mathbf{j}^{\iota \alpha}+\mathcal{I}^{\iota \alpha}+\mathcal{R}^{\iota \alpha}+\mathcal{S}^{\iota \alpha}
$$

where $t$ is time, $\theta$ is a volume fraction, $\rho$ is density, $\omega$ is a mass fraction, $\mathbf{v}$ is a mean pore fluid flow velocity vector, $\mathbf{j}$ represents non-advective transport, $\mathcal{I}$ represents interphase mass exchange, $\mathcal{R}$ represents biological or chemical reactions and $\mathcal{S}$ represents a mass source. The superscripts $\iota$ and $\alpha$ are species and phase indices, respectively.

The following identities apply to eqn (4)

$$
\sum_{\alpha} \theta^{\alpha}=1, \quad \sum_{\alpha} \mathcal{I}^{\iota \alpha}=0, \quad \sum_{\iota} \omega^{\iota \alpha}=1, \quad \sum_{\iota} \mathbf{j}^{\iota \alpha}=0, \quad \sum_{\iota} \mathcal{R}^{\iota \alpha}=0 .
$$

We also define

$$
\sum_{\iota} \mathcal{I}^{\iota \alpha}=\mathcal{I}^{\alpha}, \quad \sum_{\iota} \mathcal{S}^{\iota \alpha}=\mathcal{S}^{\alpha}
$$

Eqn (4) may be used along with eqns (5) and (6) to derive a species-summed $\alpha$-phase mass balance equation

$$
\frac{\partial}{\partial t}\left(\theta^{\alpha} \rho^{\alpha}\right)=-\nabla \cdot\left(\theta^{\alpha} \rho^{\alpha} \mathbf{v}^{\alpha}\right)+\mathcal{I}^{\alpha}+\mathcal{S}^{\alpha}
$$

Darcy's law is used to approximate momentum conservation:

$$
\theta^{\alpha} \mathbf{v}^{\alpha}=\mathbf{q}^{\alpha}=-\frac{\mathbf{k}^{\alpha}}{\mu^{\alpha}} \cdot\left(\nabla p^{\alpha}+\rho^{\alpha} g \nabla z\right)
$$


where $\mathbf{q}$ is the Darcy velocity, or volumetric flux; $\mathbf{k}$ is the effective permeability tensor; $\mu$ is the dynamic viscosity; $p$ is the fluid pressure; $g$ is the magnitude of gravitational acceleration, which is assumed to be oriented in the -k direction, and $z$ is a spatial coordinate oriented aligned with $\mathbf{k}$.

Complete specification of a physical model requires selection of a set of phases and chemical species; closure relations for interphase mass exchange and reactions; equations of state for fluid and solid phases; functions describing fluid pressure interrelationships, saturations, and conductivities; and auxiliary conditions [101]. Variations in choices among even standard models can lead to a large number of specific models, which will not be summarized in detail in this work.

Relatively little work has been done to solve optimization problems for the general class of multiphase models formulated above, due to the computational effort needed to solve such models. All but a few of the applications listed in Table 1 involve solving saturated flow and/or transport models. This class of models can be formulated for fluid flow by

$$
S_{s} \frac{\partial h}{\partial t}=\nabla \cdot(\mathbf{K} \cdot \nabla h)+\overline{\mathcal{S}}
$$

and for species transport by

$$
\frac{\partial\left(\theta^{a} C^{\iota}\right)}{\partial t}=\nabla \cdot\left(\theta^{a} \mathbf{D}^{\iota} \cdot \nabla C^{\iota}\right)-\nabla \cdot\left(\mathbf{q} C^{\iota}\right)+\mathcal{I}^{\iota}+\mathcal{R}^{\iota}+\mathcal{S}^{\iota}
$$

where $S_{s}$ is a specific storage coefficient, $h$ is hydraulic head, $\mathbf{K}$ is a hydraulic conductivity tensor, $\overline{\mathcal{S}}$ is a fluid source term, $C^{\iota}$ is the aqueous phase concentration of species $\iota, \mathbf{D}$ is a hydrodynamic dispersion tensor, $\mathcal{I}^{\iota}$ represents interphase mass transfer relations, $\mathcal{R}^{\iota}$ represents biogeochemical reactions, and $\mathcal{S}^{\iota}$ represents a source of mass. Closure relations for $\mathcal{I}^{\iota}$ and $\mathcal{R}^{\iota}$ require further specification and can assume many forms. The classic dispersion tensor is written as [22]

$$
\mathbf{D}^{\iota}=D_{i j}^{\iota}=\delta_{i j} \alpha_{t}|\mathbf{v}|+\left(\alpha_{l}-\alpha_{t}\right) \frac{v_{i} v_{j}}{|\mathbf{v}|}+\delta_{i j} \tau D^{\iota *}
$$

where $\alpha_{l}$ and $\alpha_{t}$ are the longitudinal and transverse dispersivities, respectively, $\tau$ is the tortuosity of the porous medium, and $D^{\iota *}$ is the free liquid diffusivity of species $\iota$.

The deficiencies associated with the model represented by eqn (9) and eqn (10) result from unresolved and spatially variable K. Although these deficiencies are well-known [48, 36, 61], many issues remain regarding a satisfactory solution to unresolved variability, especially for reactive systems. 
The typical set of decision variables, $n, Q_{i}$, and $\mathbf{x}_{i}$, usually enter through specification of $\overline{\mathcal{S}}$ and $\mathcal{S}^{\iota}$, which provide a mechanistic link to the state variables, e.g.

$$
\begin{aligned}
\int_{\Omega} \overline{\mathcal{S}}(t) \mathrm{d} \Omega & =\sum_{i=1}^{n} Q_{i}\left(\mathbf{x}_{i}, t\right) \\
\int_{\Omega} \mathcal{S}^{\iota}(t) \mathrm{d} \Omega & =\sum_{i=1}^{n} Q_{i}\left(\mathbf{x}_{i}, t\right) C_{i}^{\iota}\left(\mathbf{x}_{i}, t\right)
\end{aligned}
$$

where $\Omega$ is the domain of the system; $Q_{i}$ is a volumetric rate of extraction or injection and $C_{i}^{\iota}$ is a concentration of the fluid added to or removed from the system. Note that $Q_{i}<0$ indicates extraction and $Q_{i}>0$ indicates injection.

The primary state variables in eqns (9) and (10) are $h$ and $C^{\iota}$. One of the most significant differences between $\mathrm{HC}$ and PAT optimization efforts is that $\mathrm{HC}$ only requires solution of (9) for $h$, whereas PAT requires the solution of eqns (9) and (10) for $h$ and $C^{\iota}$. The solution of eqn (10) is considerably more computationally demanding than eqn (9)and requires knowledge of additional parameters and auxiliary conditions that are likely to be uncertain.

Numerical approximations are usually required to solve eqns (9) and (10). Since a typical problem involves thousands to millions of unknowns and eqns (9) and (10) often pose numerical difficulties, the solution of the governing equations is usually the most computationally intensive portion of the optimization problem. This expense leads to the frequent use of simplified models and relatively coarse discretization in space and time. Common simplification include two-spatial-dimensional models, steady-state conditions, confined aquifers, simple reaction models, single species, and local equilibrium interphase mass transfer.

Eqns (9) and (10) also contain several parameters that may be uncertain in a typical field situation, notably $\mathbf{K}$ and $\mathbf{D}$. Furthermore, the auxiliary conditions accompanying eqns (9) and (10) are often uncertain. When uncertainty in model parameters and/or auxiliary conditions exist, the model results are uncertain, or stochastic, as well. Rigorous treatment of such cases requires the solution of a stochastic optimization problem; this has rarely been accomplished in the subsurface flow and transport field [23, 13, 123, 138, 87, 121, 76].

One difficulty in comparing different works on subsurface remediation optimization is the wide range of flow and transport simulators used. The simulators differ in terms of the applied numerical method, discretization, boundary conditions, and sources and sinks. The result is that simulation outputs from a set of simulators can vary, perhaps significantly, and different values of the state variables are sent to the objective function(s) and constraint evaluations. 
Assessments of the numerical accuracy of the simulations performed during the optimization are rarely reported in the subsurface remediation literature.

Without careful assessment of the simulator accuracy, an optimization solution method could suggest a value for the decision variables that would exceed the numerical abilities of the simulator. For example, a high extraction rate could result in local velocities that would exceed grid-based numerical accuracy criteria (e.g. Peclet or Courant number constraints for finite-difference or finite-element based transport simulators). Thus, a set of decision variables may be feasible from the standpoint of the optimization criteria but infeasible due to the limitations of the flow and transport simulator. This is an example of a "hidden" constraint.

\subsection{Objective Functions}

The majority of the applications listed in Table 1 incorporate a single objective function, where the objective is to minimize cost. The remaining design goals are formulated into constraints; these are described in the following section. The literature varies widely in the definition of the cost objective function, but the function usually is divided into capital and operating costs. The capital costs of the design often depends on an integer quantity, such as the number of wells $(n)$. In the case where $n$ is a decision variable (as opposed to fixing the number of wells), the optimization problem is mixed-integer continuous. Applications that consider integer terms are designated with an I in Table 1. Various approaches have been adopted to solve this type of problem, such as formulating the optimization problem as a mixed-integer linear or nonlinear program $[122,99]$ or by approximating the capital costs as a constant multiplied by a penalty function $[82,99]$. Solving mixed-integer problems is straightforward with evolutionary algorithms [97].

The operational costs are functions of continuous decision variables (e.g. $Q_{i}$ ) as well as continuous state variables (e.g. $h$ and $C^{\iota}$ ). These functional definitions are often nonlinear. Applications with nonlinear objective functions are designated as NL or DYNL in Table 1. For example, the cost of groundwater extraction is a nonlinear function of the extraction rate $Q_{i}$ and hydraulic head $h[2,141]$. In the case where $h$ is included in the extraction cost term, the hydraulic head is estimated via a groundwater flow simulator based on eqn (9), which is usually solved numerically. In the special case of a confined groundwater aquifer, the hydraulic head is linearly related to the extraction or injection rate decision variables. In this case, linear programming can be used to obtain the solution [2]. In addition, repeated, computationally expensive solutions of eqn (9) are unnecessary, since superposition can be employed [141]. However, if the aquifer must be treated as unconfined, $h$ is a nonlinear function 
of the pumping rate decision variable, $Q_{i}$, and neither linear programming nor superposition are applicable [72].

Treatment of extracted groundwater is performed with a range of technologies, such as granular activated carbon adsorption (GAC), air stripping, and chemical or thermal oxidation. The installation and operational costs associated with these technologies depend on the influent flow rates and concentrations. For example, the operational cost for GAC is a nonlinear function of concentration $\left(C^{\iota}\right)$ and a linear function of influent flow rate [47]. In the case where $C^{\iota}$ is included in the objective function, the concentration field must be simulated. If the choice of treatment technology is unspecified and becomes a decision variable in the optimization problem, then categorical variables are involved.

As mentioned previously, the formulations of the cost objective functions vary from work to work. In some cases, variations in the cost formulations are related to differences in the engineering problem to be solved (e.g. HC vs. PAT). But, in other cases, the choice of formulation seems to be arbitrary. This arbitrariness is problematic, since it makes it difficult to compare one work to another, and it implies that there is no consensus regarding the engineering problem to be solved. Several researchers have shown that the optimal solution is sensitive to the cost formulation [43, 31, 99], but a systematic study of the effects of cost function formulation on the optimal design has not been performed. In addition, cost objective functions for other remediation technologies have been reported only rarely.

\subsection{Constraints}

Constraints, $\Omega_{z}$, are placed on decision variables (designated as D in Table 1) and/or state variables (designated as LS or NLS in Table 1). The decision variable constraints are usually associated with a technological limitation, such as the maximum extraction rate in a well or the total flow rate associated with all wells. In remediation applications, constraints on state variables are associated with the goal of the remediation effort. For example, the constraints for $\mathrm{HC}$ require that the hydraulic gradients and corresponding velocity vectors in the area comprising the plume be oriented towards the extraction wells. Mulligan and Ahlfeld [106] and Ahlfeld and Mulligan [2] noted that the hydraulic gradient approach for enforcing the capture constraint is attractive because the constraint is linear with respect to the state variable, $h$. They note that, in practice, however, inefficient designs can result unless considerable effort is expended in calibrating the constraint.

Alternatively, the capture constraint is based explicitly on whether the con- 
taminant is captured by an extraction well, referred to as an advective control constraint. In this case, the contaminant mass is represented as a series of particles. The velocity field for each particle is determined by Darcy's law, eqn (8). Solution of eqns (8) and (9) provides streamlines that define the pathline of each particle. The constraint is expressed such that particles tracked from the edge of the contaminant plume are required to terminate at an extraction well. The difficulty introduced by considering advective control constraints is that the constraints are nonlinearly related to extraction rates. Advantages and disadvantages of various formulations of the advective control constraint are discussed by Mulligan and Ahlfeld [106].

In the case of PAT optimization, constraints are incorporated that explicitly consider contaminant mass reduction. Constraints based on contaminant mass reduction are based either on values of contaminant concentration at observation points or global contaminant mass. The introduction of contaminant constraints increases the complexity of the problem considerably. First, the constraint is a nonlinear function of the decision variables (extraction or injection rates). This nonlinear relationship introduces local minima and non-convexity in the envelope surrounding the feasible region [11]. Second, developing and executing a contaminant transport simulator requires a significant amount of human and computational effort, especially when compared to the effort involved in groundwater flow simulation.

\subsection{Solution Approaches}

The ideal solution method is capable of converging to the global minimum while minimizing the computational effort. The appropriate class of methods for solving the optimization problem depends on the problem formulation, i.e., the form of the objective function and constraints. Table 1 identifies the solution approaches taken for each application, which range from the well established linear programming methods (LP) to the newer global optimization methods (GO). Here, we briefly review optimizations methods that have been applied to PAT design, since optimization of PAT design has, in general, proven more difficult than HC design. Since PAT formulations include constraints based on contaminant mass reduction, nonlinear optimization methods are needed to solve the PAT design problem. The most frequently applied nonlinear optimization methods are derivative-based, nonlinear programming methods (NLP), such as reduced gradient methods (e.g. MINOS, cf [71, 99], or sequential quadratic programming, (SQP, e.g. NPSOL, cf [74]), or the successive linear quadratic regulator method [31]. Direct search (DS) methods, such as modified simplex, Nelder-Mead simplex, or parallel directive search have been applied far less frequently [144]. 
The derivative-based methods have been criticized with regard to their ability to handle the non-smooth objective functions, local minima, and nonconvexity that are typical in problems where contaminant mass reduction is a constraint. In response, the so-called global optimization (GO) methods have been applied to solve the PAT optimization problem. For example, in [12], MINOS [107], a standard NLP code, is trapped in local minima in some of the results, leading to a preference for a genetic algorithm, one of the classes of GO methods.

A GO method attempts to find the global minimum and is designed to that end. Methods such as genetic algorithms, simulated annealing, and DIRECT are global optimization methods. A true global optimization method must, in theory, examine a dense mesh in the design space [129]. Therefore implementations rely on heuristics. This class of methods includes outer approximation methods $[82,83]$, where the optimal solution is found by making successive "cuts" in the objective function space, along the feasible space boundary.

The evolutionary algorithms (EA's) are also classified as GO methods. EA's have the advantage of straightforward incorporation of discontinuous decision variables and relatively straightforward implementation on parallel processor computing platforms. EAs applied to PAT design optimization include variants of genetic algorithms, or GA's, [116, 118, 79, 12], simulated annealing [93, 117], and tabu search [147]. However, EA's are criticized because the performance of the algorithms depends on values selected for heuristic parameters. For example, the simple GA is controlled by population size, crossover rates, and mutation rates. In most $\mathrm{EA}$ applications that have considered remediation optimization, the optimal parameter values have been determined by trial and error. Recent work by Reed et al. [115] and Erickson et al. [51] has resulted in guidelines for selecting optimal parameter values associated with variants of the GA.

Only a few extant works compare the performance of solution methods. Karatzas and Pinder [83] compared GO (outer approximation) to NLP (MINOS) for determining optimal pumping rates for 40 wells with respect to cost, given maximum pumping rate and maximum concentration constraints. They found that the outer approximation technique provided a significantly less costly solution for a fraction (1/15) of the computational effort. Yoon and Shoemaker [144] compared EA, DS, and NLP methods for a bioremediation problem with time-varying extraction rates. The successive approximation linear quadratic regulator (SALQR) technique, an NLP method, was found to require considerably less computational effort than the other methods. However, we note that SALQR has a limitation in that it cannot be used to solve discontinuous problems with multiple local optima.

Aly and Peralta [12] compared EA (simple GA) with NLP (MINOS) and 
a combination of NLP and integer programming to determine optimal, timevarying pumping rates. Two different formulations were tested: minimizing the maximum concentration remaining with cost as a constraint and minimizing cost with concentration remaining as a constraint. For both formulations, the GA performed as well or better than NLP-based optimization in terms of minimizing the objective function values. However, no information was given to compare the computational effort among the different methods.

A more subtle issue is that there is no guarantee that the objective function will even return a value, for example an internal iteration within a PDE solver may not converge. Failure of the function evaluation has been observed in practice $[142,34,30,42,27]$. We call the need for the function to return a value a "hidden constraint" because only an attempt to evaluate $f(z)$ can determine if $z$ is a feasible point. Such constraints are also called "virtual constraints" [42] or "yes/no" constraints [29]. In some cases [34, 32, 52], it is even difficult to find a point to begin an iteration.

\subsection{Multi-Objective Optimization}

The need for multi-objective optimization stems from the observation that decisions regarding remediation design are frequently based on multiple, conflicting criteria [56]. Yet, as indicated in Table 1, the vast majority of subsurface remediation optimization applications have been based on achieving a single objective: minimizing costs. Additional objectives could be considered, such as maximizing cleanup performance, maximizing reliability, and minimizing cleanup time. These objectives are incorporated implicitly into single objective optimization frameworks by transforming them into fixed constraints. The MO approach allows the decision-maker complete flexibility and the opportunity to view tradeoffs between objectives. However, the number of choices offered in the problem solution may be overwhelming; furthermore, solving MO problems may require complex and computationally expensive solution methods.

The potential value of viewing tradeoffs between multiple criteria is significant. First, plots of the curve could reveal areas where tradeoffs between conflicting objectives are uneven, and thus represent inefficient values of the decision variables. For example, a cost vs. cleanup performance curve might indicate that a threshold contaminant concentration exists where the slope of the curve changes from linear to exponential. Second, tradeoff curves could be used to prioritize among sites; a decision maker could use multiple tradeoff curves to intelligently allocate resources for maximizing risk reduction across a set of sites. These hypothetical examples demonstrate that the application of multiobjective optimization to subsurface remediation design could also have a 
significant impact on decision-making and policy.

The generic multi-objective problem can be stated as

$$
\min _{\mathbf{z} \in \Omega_{z}} f(\mathbf{z})=\min _{\mathbf{z} \in \Omega_{z}} f\left(f_{1}, \ldots, f_{n_{k}}\right)
$$

where $f$ is a multi-objective functional depending upon the vector of $n_{k}$ objective functions $f_{k}$.

More than one set of decision variables, w, and corresponding functional evaluations of $f_{k}$ can satisfy eqn (14). In Figure 1, we have plotted a few hypothetical evaluations in a two-objective function problem $\left(f_{1}\right.$ and $\left.f_{2}\right)$. Each point represents the evaluation of $f_{1}$ and $f_{2}$ that corresponding to a given value of the decision variable, $w_{1}, w_{2}, w_{3}$. The concept of "Pareto optimality," states that one candidate dominates another only if it is at least equal in all objectives and superior in at least one. For example, in Figure 1, where the objective is to minimize both objective functions, designs $w_{1}$ and $w_{2}$ dominate design $w_{3}$ because they are superior in both objectives. Moreover, designs $w_{1}$ and $w_{2}$ are said to be non-dominated.

From this simple example, we can see that the concept of Pareto optimality gives rise to a set of solutions known as the Pareto optimal set. The $K$-dimensional surface defined by the non-dominated solutions is known as the Pareto front, or tradeoff surface. A class of multi-objective EA's (known as EMO's) that operates on the concept of Pareto optimality. Evolutionary methods are particularly suitable for solving multi-objective problems, since they deal simultaneously with a set of possible solutions [40]. The class of EMO's includes the multi-objective genetic algorithm (MOGA, [e.g. 53]), vector-evaluated genetic algorithm (VEGA [124]) and the Pareto genetic algorithm (PGA, [69]). Ritzel et al. [116] applied PGA and VEGA to a HC optimization problem where the objectives were to minimize the containment design cost while maximizing the design's reliability. Ritzel et al. [116] concluded that the PGA was superior to the VEGA in finding the largest portion of Pareto optimal solutions.

Rogers and Johnson [119] implemented a multi-objective simulated annealing algorithm (SAA) using a linearly weighted formulation along the lines of eqn (15). The objective functions were "minimize source removal cost" vs. "minimize source remaining." Although the weights were fixed, the stochastic nature of the SAA provided a range of solutions with variable values of the objective function. These solutions were used to plot a tradeoff curve. Erickson et al. [51] used the niched Pareto GA (NPGA) developed by Horn [78] to develop tradeoff curves for cost vs. contaminant mass remaining for PAT problems. The NPGA was compared to a single objective GA, where the contaminant mass remaining was fixed as a series of constraints, and to a random enumer- 
ation. The NPGA was shown to be superior in terms of both computational performance and Pareto front metrics for larger numbers of decision variables (e.g. $\mathrm{n} \geq 5$ extraction wells).

The challenge remains for the decision-maker to choose a "best" solution among the infinite number of solutions on the tradeoff surface. If the relative importance (to the decision-maker) of each objective function is known a priori, we can aggregate the objectives into a single objective function and solve the resulting problem with the appropriate optimization method. For example, we could minimize a weighted, linear combination of the objective functions, as in

$$
\min _{\mathbf{z} \in \Omega_{z}} f=\min _{\mathbf{z} \in \Omega_{z}} \sum_{k=1}^{n_{k}} a_{k} f_{k}
$$

where $a_{k} \geq 0$ are weighting coefficients representing the relative importance of the $n_{k}$ objective functions. The usual approach is to properly scale the objective functions and to scale the weighting coefficients as $\sum_{k=1}^{n_{k}} a_{k}=1$. This approach was used by Xiang et al. [143] in a two-objective PAT design problem, where the weights were chosen as $a_{1}=a_{2}=0.5$, implying equal importance to the objective functions. In cases where the values of $a_{k}$ are unknown a priori, it is necessary to solve eqn (15) for many values of $a_{k}$. Each solution can be plotted on a tradeoff surface; however this approach is limited to finding solutions only where the tradeoff surface is convex.

Fig. 1. Pareto domination 


\subsection{Stochastic Approaches}

Subsurface design problems are usually solved deterministically, as seen in the majority of the works cited in Table 1 . However, it has been noted for more than a decade that, due to the variability of and impossibility to characterize subsurface systems, stochastic modeling approaches are more appropriate for many subsurface problems $[137,138,14,87]$. Of primary concern is the spatially variable nature of hydraulic conductivity, which is well known to vary markedly in space and is usually assumed to be log-normally distributed $[55,138,87]$. However, hydraulic conductivity is not the only aspect of subsurface models that is variable and difficult to characterize; boundary conditions, initial conditions, mass transfer parameters $[112,114]$, reaction rate coefficients [103], and even the form of the flow and transport model itself $[84,87]$ are often uncertain. Because the unknown model parameters are distributed in space, inverse solution approaches are notoriously ill posed [84]. Regardless of the difficulty associated with problems of concern, this situation is realistic, and efforts to incorporate uncertainty into the design of subsurface management solutions are critical for advancing a solution to the true problem of concern.

In a series of papers, Freeze and co-workers advanced a framework for hydrogeological decision analysis [58], considered a simple subset of the general framework that relied upon simple analytical models [94], extended the work to uncertainty in a variably saturated model using Monte Carlo analysis to assess slope stability [127], and investigated the important question of the value of information [57]. This comprehensive approach has yet to be fully exploited due to technical difficulties associated with the variety of coupled problems involved: parameter identification, stochastic flow and transport modeling, chance constraints, multi-objective problems, value of information, and riskcost-benefit modeling. Interest in this conceptual approach remains high and recent work on a PAT application has shown the importance of an accurate characterization of nature and probability of system failure [121].

There remains a divide between practitioners charged with remediating subsurface systems and researchers focused on advancing improved techniques to optimize the design of such systems. This is largely due to the difficulty with the real problems of concern and the need to tackle small bites of the complex problem to incrementally advance the field with a manageable level of effort. This conflict leaves practitioners unsatisfied and the real problems largely unsolved. Practitioners may view the problem as an optimal control problem and implicitly acknowledge that they are uncertain about the system behavior - relying on measurements of system performance with time to inform their decision making. 
The work of Lee and Kitanidis [87] is useful from this perspective. They formulated the problem as a stochastic optimal control problem in which sequential adjustments to pumping rates were made based upon all prior information, which was used to update the model predictions using an extended Kalman filter. Such approaches can be termed stochastic control problems with recourse. They showed that such approaches that naturally account for uncertainty in parameter distributions and modeling errors provide a more robust solution than a sequential deterministic management plan. Lee and Kitanidis [87] did not couple this approach with the details of the optimal design, only time varying pumping rates, and they did not consider the value of information or complex and realistic patterns of subsurface heterogeneity. Bear and Sun [23] have also recently advanced a multistage recourse approach considering design reliability.

Reliability is a natural and important aspect of remediation design that can be considered using stochastic approaches and has been considered in recent works $[134,23,13]$. Because of the links among cost, reliability, and uncertainty, this is a natural case in which multi-objective approaches are useful. This is so because highly reliable solutions can be relatively very expensive and presenting a decision maker with this sort of information a priori might well effect the decision made.

\subsection{Challenges}

The many challenges in the optimization of subsurface remediation problems include contending with objective functions and constraints that are nonlinear with respect to decision and state variables, state variables that are stochastic, the numerical solution of difficult governing equations, and multiple objectives. In light of these challenges, we believe that the community should consider undertaking the following tasks:

(1) introduce more efficient and robust optimization solution methods,

(2) develop a set of test problems for the purposes of benchmarking,

(3) consider a wider range of remediation technologies,

(4) consider routinely the use of stochastic representations of subsurface properties,

(5) represent more realistically the physics, chemistry, and biology in the contaminated subsurface,

(6) develop more efficient flow and transport simulators, and

(7) apply multi-objective solution methods. 


\section{Potential Methods}

In this section, we discuss emerging ideas for meeting the challenges in the optimization of subsurface flow and transport problems. We begin with a holistic view of the optimization problem and introduce the "all-at-once" approach, where decision and state variables are considered simultaneously. We next consider a class of optimization solution methods: deterministic sampling methods. These methods have received scant attention in the subsurface optimization community, but show promise for handling the typical difficulties (e.g. local minima, nonconvexity, hidden constraints, and nonsmoothness) encountered in subsurface flow and transport optimization problems. We address potential approaches for dealing with stochastic optimization problems, which we anticipate will become the norm, rather than the exception, in the near future. Lastly, we discuss potential applications of multi-objective optimization methods to subsurface optimization problems.

\subsection{Formulation of Optimization Problems}

Having selected appropriate algorithms, one still must decide on how the state and design variables will be treated in the optimization. The all-at-once approach treats both $w$ and $u$ as optimization variables. This means that the optimization looks at the vector $\mathbf{z}=(\mathbf{w}, \mathbf{u})$ as a single set of design variables, ignoring the dependence of $\mathbf{u}$ upon $\mathbf{w}$. In this approach, the simulator and optimization code must be tightly coupled, since no distinction is made between state and decision variables in the optimization algorithm. This has the significant advantage that if the optimization method and the simulator are designed simultaneously, one can often compute gradient information from linear sensitivity equations [85] that can be solved as the simulation runs. Such equations can be derived directly, with an adjoint formulation, or with automatic differentiation [73].

One disadvantage of the all-at-once approach is that it is difficult to use an existing simulator in this mode because (a) differentiating the mathematical model with respect to both decision and state variables requires access to parts of the code that may not be intended for user access, and (b) the simulator may not be designed with optimization in mind, having, for example, control structures (if-then-else and case statements, for example) that add artificial nonsmoothness. While a global optimization algorithm method can, in principle, overcome this problem, the high cost in function evaluations, especially for large numbers of design variables, may be overwhelming.

If the problem is smooth and has only continuous variables, the all-at-once 
approach makes nonlinear constraints on $\mathbf{w}$ much easier for the optimizer to handle. This method for handling state constraints is well-known in the control literature [25], where very large problems have been solved.

In $[46,104]$ and related papers, a differential-dynamic programming [96] method has been applied to PAT and BIO problems. The simulator is tightly coupled with the optimizer, computing gradients and second derivatives in a way similar to the method of adjoints in optimal control. This means that some derivatives must be available analytically to form the adjoint equations. The method is equivalent to a Newton iteration and, therefore, converges rapidly for smooth problems. The simulator must be designed at a detailed level to work well with this approach. See $[44,91]$ for examples of the these simulators.

The level of effort in an all-at-once approach is high. Experts in optimization, groundwater modeling, and simulation must combine in the design of a stateof-the-art simulator that can interface at a low level with more than one optimization algorithm. In many cases, however, one must use a simulator that was not necessarily designed with optimization in mind and is difficult to modify. The "black box" approach treats w alone as the optimization variable. With this approach, any optimization method can be applied and simulators can easily be changed, even when the optimization is in progress. One scenario for the black box approach is if stand-alone flow and transport simulators are combined to track contaminant transport, producing an objective function value that is given to the optimizer. The flow, transport, and optimization codes can be completely arbitrary and need not be developed simultaneously. Moreover, the flow and transport models can be changed as the optimization progresses.

\subsection{Deterministic Sampling Methods}

Sampling methods for solving optimization problems have been developed primarily over the last decade in the applied mathematics community, but rarely have been applied to subsurface flow and transport problems. Sampling methods avoid local minima by examining the function at arrays of points, adjusting the size of the array in response to the progress of the optimization. In this way local minima can be "stepped over" when the points in the array are widely spaced and, as the optimization continues and the spacing becomes tighter, global minima can be accurately resolved. Sampling algorithms have performed well for problems with multiple local minima in a variety of applications, [86, 28, 21, 142, 131]. Battermann et al. [21] solved a problem in control of subsurface temperature with such a sampling method.

Many problems with multiple local minima, nonconvexity, hidden constraints, 
and nonsmoothness can be most efficiently addressed with sampling methods. Unlike simulated annealing or genetic/evolutionary methods, we consider methods which are specifically designed to solve problems with difficult, but not violently oscillatory, optimization landscapes, such as those in Figure 2.

Figure 2 (a) $[131,67]$ is a graph of the negative of the maximum power-added efficiency of an amplifier as a function of the real and imaginary parts of the second harmonic load impedance. The simulator for this work had internal iterations that required varying numbers of iterations away from the optimal point, leading to the rough surface at the peak. The model smoothed out near the optimal point.

Figure 2 (b) [21] is a graph of the response of a simulation of subsurface temperature to the pumping rates of two control wells. The nonsmoothness comes from the thermal dispersion tensor in the flow and temperature simulation. There are no internal iterations in the simulator, and therefore the surface is smoother than that in Figure 2 (a).

Fig. 2. Optimization Landscapes

(a) Semiconductor Modeling

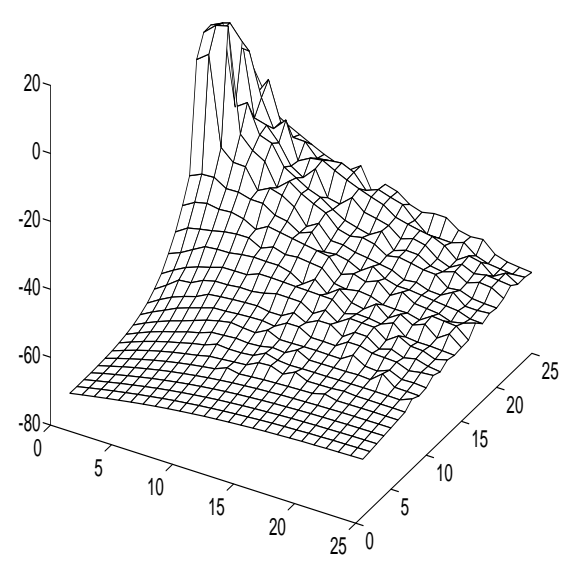

(b) Subsurface Temperature

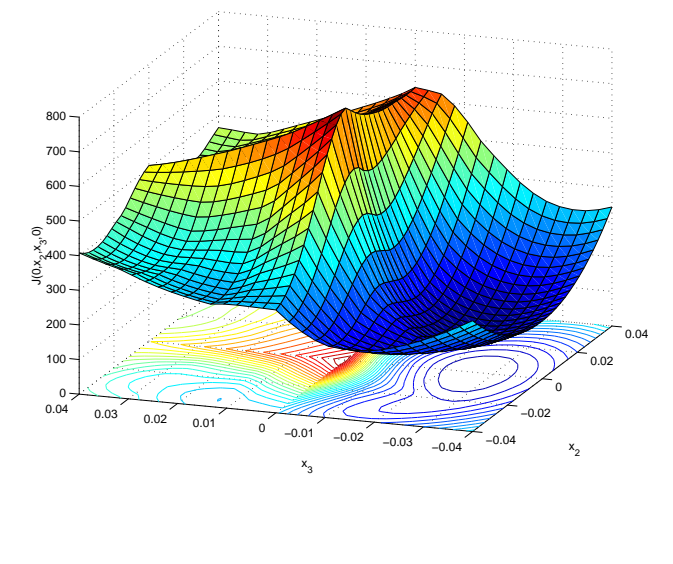

By a sampling algorithm we mean one which uses only evaluations of the function to be minimized in the optimization. Sampling methods converge slowly, and when gradient information is available, conventional methods perform far better at resolving local minima to high precision. Therefore, sampling methods are usually applied to difficult problems with complex optimization landscape and to problems for which derivative information is not available $[131,54,68,28,86,27]$. 
One can improve coordinate search by changing either the stencil or by using the stencil values in more subtle ways. Methods like Hooke-Jeeves [77] or MDS only examine the stencil until a better point is found and then either move to that point (MDS) or use the new, better, point as a base for further exploration of the search space. Implicit filtering [85, 67] and Hooke-Jeeves also use the values of $f$ at the stencil points to look for new, promising, points to evaluate $f$. In the case of implicit filtering, the new point may not be on the current grid.

When applied to smooth functions or small perturbations of smooth functions, stencil-based methods are supported by a rich convergence theory [85, $135,136,67,35,17,15]$. The results in Audet and Dennis [18] require only smoothness at a limit point to prove that that limit point is stationary in a generalized sense. In practice these methods perform well for problems far more difficult than those covered by the theory. These methods have been applied to problems with both hidden $[42,28,34]$ and explicit $[88,16,15]$ constrains. New methods for problems with categorical variables have all been developed $[17,86]$.

Two examples of stencil-based methods are implicit filtering and the mixedvariable-programming method. Implicit filtering $[67,85,33]$ is a finite-difference projected quasi-Newton algorithm in which the difference increment is varied as the optimization progresses. In this way local minima that are artifacts of low-amplitude noise do not trap the iteration and the noise is implicitly filtered out. The method was designed for problems with objective functions that are small perturbations of smooth functions.

The MVP (Mixed Variable Programming) method [17] is an approach to categorical variables that extends the pattern in the categorical variables by using application-specific knowledge about what "nearby" means for the categorical variables. If we were attacking convex problems so that a global solution was a reasonable goal, then even this would not be required. The user notion of "nearby" is used to put a metric on the mixed space of continuous and categorical variables and hence define a local optimizer. In this way the search and can be managed in a similar way as for the continuous variable case, and any method can be used to obtain improvement in the continuous variables. A simple pattern search method of the type described in Torczon [136] was used to improve the continuous variables in an application [86].

Exceptions to the stencil-based paradigm are the Nelder-Mead [109] and DIRECT [81] methods, where the sampling is less structured. The Nelder-Mead algorithm adjusts the shape of the sampling pattern to respond to the relative values of the best few points. In this way the method seeks to follow paths of decreasing function values. When working well, the Nelder-Mead method is very effective. However, it can fail on very easy, smooth problems [85]. 
The DIRECT (DIviding RECtangles) method partitions the design space into hyperrectangles, sampling at the centers, and subdividing those rectangles that, based on their size and the function values at the centers, show promise. The rules for subdivision vary $[81,59,60,19,20]$ depending on the number of the design variables and the bias toward local or global search.

\subsection{Stochastic Solution Methods}

Routine use of stochastic solution approaches will be necessary to produce mature solutions to many subsurface design problems. This is so because of the inherently spatially variable nature of subsurface systems. While we advocate approaches such as the comprehensive coupled approach of Freeze and co-workers $[58,94,127,57,121]$, significant challenges remain that will require new approaches. Chief among these are alternatives to expensive highresolution Monte Carlo solutions to assess the effect of uncertainty in model parameters on model predictions. While relatively few Monte Carlo simulations have provided useful information in some cases $[134,13]$, accurate representation of high reliability designs requires high resolution simulations to resolve the tails of the probability density function. Such high reliabilities are of interest if the penalty for non-compliance is high [121]. However, Smalley et al. [126] used a noisy genetic algorithm to show that this is not necessarily the case.

Alternatives to Monte Carlo analysis are possible [87], but resolution remains an issue. For example, evolving analytical methods for assessing the crosscovariance between hydraulic conductivity and heads [38, 39, 111, 37] provide possible alternative approaches. Another interesting notion worth exploring is the use of stochastic finite element approaches, which provide a means to assess the probability density function of the model output in terms of the probability density function of model input parameters using series expansions for both model inputs and outputs. When effective, such approaches can greatly reduce the computational effort needed compared to Monte Carlo methods. These methods have received relatively little attention in the water resources literature $[65,64,63]$. Thus, the ability of these methods to adequately resolve model output tails from a density function remain an open issue.

Another stochastic optimization approach, second order cone optimization (SOCO) [24], has been used recently by Ndambuki et al. [108] to consider the effects of uncertainty on optimal groundwater management policies. SOCO transforms the stochastic optimization problem into uncertainty ellipsoids that are functions of the uncertain parameters (in this case, the transmissivity field). Ndambuki et al. [108] formulated a multi-objective problem where the objective of maximizing pumping rates in a water supply system was pitted 
against maximizing reliability of the design. Solutions were obtained using the multiple scenario approach (a variant of the Monte Carlo approach) and SOCO and it was found that SOCO was more robust for a given number of scenarios. It was also noted that SOCO works especially well when recourse is considered, since SOCO requires that only a relatively small part of the problem must be re-executed when new parameter information is provided. Drawbacks of SOCO are that the method requires convex objective function surfaces and linear (or linearized) relationships between the decision variables and the uncertain parameters.

Evolving approaches, such as the Bayesian Maximum Entropy (BME) approach $[36,26,125]$, provide a means to incorporate multiple source of hard and soft information and physical laws in an information processing framework that is not restricted to traditional Gaussian approximations [138, 87]. The BME method is well-suited to the complex, multistage information processing task needed to advance more effective design approaches in the light of uncertainty, but much work remains to be done to realize this potential.

Regardless of the numerical solution approach used, high-resolution distributed parameter models will continue to stretch our available computational resources for the foreseeable future. It will be necessary to take advantage of the state-of-the-art computational facilities to affect the most satisfying solutions. Currently, this means massively parallel, distributed memory computers. While it may be trivial to get high performance for a Monte Carlo-based approach on such a computational architecture, this situation is certainly not the case for individual model simulations and evolving analytical and semianalytical methods.

\subsection{Multi-objective Solution Methods}

Evolutionary algorithms are attractive for solving multi-objective problems, but at least three challenges remain to be resolved. First, EMO's can be computationally burdensome, due to the requirement of performing many (hundreds to more than tens of thousands) of objective function evaluations. This problem can be ameliorated by exploiting the easy parallelization of the objective function evaluations, within each generation. In addition, the field of EMO's is expanding rapidly. For example, the micro-GA of Coello Coello and Pulido [41] and the recent version of the non-dominated sorting GA (NSGAII, [49]) have shown significant decreases in the computational effort required for convergence to a Pareto optimal solution. Second, the algorithms' performance depends on heuristic parameters (e.g. population size, crossover rate, niche size, etc.) for which the optimal values are unknown. Attempts are being made to resolve this problems. Reed et al. [115] and Erickson et al. [51] have 
proposed guidelines for selecting optimal values of the heuristic parameters associated with variants of the GA.

The third challenge is to develop performance metrics that can be used to define convergence and for comparisons among Pareto solutions generated by different EA's. In addition to computational efficiency, there are two general performance metrics multi-objective EA's: the Pareto optimality of the solutions and the portion of the Pareto front found by the algorithm. Since the "true" Pareto front is usually unavailable for a typical subsurface remediation problem, these metrics can only be applied in a comparative sense.

The MO approach can be expanded to include uncertainty implicitly, where the objective functions values are based on model equations $\tilde{\mathbf{g}}$ that are stochastic. In this case, the solutions to the MO problem are expressed as values of the decision variables that correspond to a set of probability density functions (pdf) for the objective functions. For example, we could formulate the problem as in the following:

$$
\begin{aligned}
& \text { find w such that } \min \tilde{f}^{\text {cost }}=\min \tilde{f}^{\text {cost }}[\mathbf{w}, \tilde{\mathbf{u}}(\tilde{\mathbf{g}}(\mathbf{w}))] \\
& \qquad \begin{aligned}
\max \tilde{f}^{\text {clean }} & =\max \tilde{f}^{\text {clean }}[\mathbf{w}, \tilde{\mathbf{u}}(\tilde{\mathbf{g}}(\mathbf{w}))] \\
& \text { and } \mathbf{u}, \mathbf{w} \in \Omega_{z}
\end{aligned}
\end{aligned}
$$

Here, the solution, w, is the vector of decision variable values that would produce a given pdf of cost $\left(\tilde{f}^{\text {cost }}\right)$ and cleanup performance $\left(\tilde{f}^{\text {clean }}\right)$ and meet the constraints. In other words, the solution gives values of the decision values where there is an associated probability that given level of cost or cleanup will occur.

This approach has been taken in Figure 3, which shows a tradeoff curve "envelope" for a PAT design problem. In this problem, we are attempting to find the least cost solutions for a given cleanup target, which is expressed as the maximum concentration remaining in the aquifer at the end of a fixed remediation period. The hypothetical problem involves applying PAT to remediate a conservative contaminant plume in a homogeneous aquifer. The aquifer hydraulic conductivity is a stochastic variable, with a uniform, logarithmic probability distribution ranging over approximately four orders of magnitude. We use steady-state groundwater flow and transient transport simulators based on eqns (9) and (10). The decision variables are the static extraction rates for two wells that are located in the center of the plume. The cost objective function includes nonlinear pumping lift costs and nonlinear treatment costs, based on treatment by granular activated carbon. More detail on the simulators, the cost objective function, the hypothetical aquifer configuration and the multi-objective optimization method can be found in Erickson et al. [51]. 
The envelopes in Figure 3 represent the 95\% confidence intervals around an expected value (bold line). The envelope was generated by randomly sampling the distribution of hydraulic conductivity values and finding the corresponding Pareto optimal sets of pumping rates, with respect to cost and contaminant mass remaining in the aquifer. One of the interesting, but not entirely unexpected, results indicated in Figure 3 is the significant increase in the width of the envelope as the cleanup target becomes more restrictive.

Fig. 3. Cost vs. mass remaining tradeoff curve for $95 \%$ most probable cost and mass remaining

Alternatively, uncertainty can enter the problem implicitly via the constraints, or explicitly, via an objective function such as

$$
\max f^{R}=\max f^{R}[\mathbf{w}, \tilde{\mathbf{u}}(\tilde{\mathbf{g}}(\mathbf{w}))]
$$

where $f^{R}$ is a measure of reliability. Reliability can measured, for example by determining the probability that a certain cleanup level, at a certain cost can be achieved. The Pareto optimal set of $\mathbf{w}$ and associated values of $f^{R}, f^{\text {cost }}$, and $f^{\text {clean }}$ would form a surface, where tradeoffs between cost, reliability, and cleanup performance could be viewed.

An alternative approach to solving for the entire Pareto front or tradeoff curve is to determine weights, or user preferences, and solve for example eqn (15). This approach has been applied to surface water optimization problems, but 
has not been applied to subsurface remediation problems to our knowledge. The weights $\mathbf{A}=\left[a_{1}, a_{2}, \ldots a_{K}\right]$, can be determined a priori by developing utility functions that describe the decision-maker's preferences with regard to tradeoffs among multiple objectives [130]. Other a priori methods include lexicographic ordering and goal programming.

Yet another approach is taken with interactive methods, which do not assume prior knowledge of decision-maker preferences, but act to guide the decision-makers towards their preferences among or ordering of objective functions. These methods are iterative and begin with some initial guess as to the decision-maker's preferences. For example, the multi-objective problem could be formulated as in (15) and the initial guess could be an equal set of weights (e.g. $a_{k}=1 / n_{k}$ for all $k$ ). The corresponding optimal solution is found (with the appropriate single-objective optimization method) and the decision-maker determines whether the solution is appropriate. If not, a new set of weights is chosen, and procedure continues until the preferable set of weights is found.

There are several interactive methods in the decision analysis literature [100]; some of these methods, such as ELECTRE [120], have been applied in multiobjective, surface water quality management problems [50]. Most of the methods are based on the concept that determining the decision-maker's preferences is an optimization problem in itself. Thus, as the preferences become better known, iteration by iteration, a value (or preference or utility) function can be fitted to the prior results and used to predict the decision-maker's final or near-final results. For example, a steepest descent method can be used to determine the step-size and direction to be taken for the weight vector ( $\left.\mathbf{A}=\left[a_{1}, a_{2}, \ldots a_{K}\right]\right)$ solution at the next iteration level (as in the GDF method, [62]). The choice of an appropriate iterative method depends on the quantitative and qualitative characteristics of the underlying optimization problem, such as the differentiability of the objective functions and the decision-maker's experience in stating their preferences among the objective functions.

\section{COMMUNITY PROBLEMS}

While resolution of the challenges in subsurface optimal design will take considerable time and effort, we believe that the community will benefit immediately from a framework for comparison among methods. In this section, we outline the general principles and details needed to define a systematic set of test problems and a basis for comparing competing methods. We advocate several general principles to guide the design of a set of community problems:

(1) the problems should be indicative of real-world problems and should cover 
a range of anticipated difficulties in finding a solution, i.e. non-convexity, non-smoothness, and multiple local minima;

(2) the problems should be specified in complete detail, allowing easy replication of the solution by any investigator;

(3) the problems should have multiple uses, including numerical and optimization method development, testing, and comparisons, and education and community training;

(4) the problem specifications should be intended to encourage, not restrict, innovation in all areas of optimal design and subsurface science; and

(5) guidelines should be developed to allow fair and accurate comparisons among competing methods.

We outline in the following a range of applications, physical models, objective functions, and constraints that together make up a set of test problems consistent with the principles outlined above. The units associated with each variable follow the $\mathrm{m} \cdot \mathrm{kg} \cdot \mathrm{s}$ system; these units can be assumed in cases where they are not specifically mentioned. In order to conserve space, complete details of all aspects of the community problems have been included in the supplemental information (SI) section of this paper (http://www.elsevier.com/locate/advwatres). We outline in the text that follows the general nature of these problems.

\subsection{Applications}

A set of four applications is listed in Table 2 along with corresponding entries for possible choices of domains, model formulations $(g)$, objective functions $(f)$, and constraints $\left(\Omega_{z}\right)$, the details of which are included in the sections that follow. The applications considered are: a well-field design, well-field design with seawater intrusion, the capture of a contaminant plume, and PAT remediation of a contaminant plume(s). The domains include a range of homogeneous and heterogeneous systems. The models include mathematical representations of fluid flow and contaminant transport in confined and unconfined aquifers. The objective of all problems is to minimize the cost of the installation and operation of a set of wells. In addition, the PAT application includes a treatment cost component. Decision variables include the number $(n)$, location $\left(x_{i}, y_{i}, z_{i} ; i=1, n\right)$, and pumping rates $\left(Q_{i}\right)$ for all wells. Constraints include bounds on individual and total pumping rates and heads.

The problem specifications are outlined so as to allow latitude in approaching the design problem. For example, well locations and numbers can be arbitrary or selected from a specified candidate set. Further, pumping rates can be taken as constant, constant over intervals, or follow some user-specified function. By leaving aspects of the problem solution to the modeler, we encourage creativity and method evolution to deal with the most general, and demanding, designs. 
Table 2

Community Application Designs

\begin{tabular}{|l|l|l|l|l|l|}
\hline Application & Description & Domains & Models & Objectives & $\begin{array}{l}\text { Constraint } \\
\text { Equations }\end{array}$ \\
\hline \hline I & well-field & A-E & $1 \& 2$ & a & $(52)-(54)$ \\
\hline II & $\begin{array}{l}\text { well-field/ sea- } \\
\text { water intrusion }\end{array}$ & A-E & 3 & a & $(52)-(55)$ \\
\hline III & capture zone & A-E & 2 & a & $\begin{array}{l}(52)-(54), \\
(56),\left({ }^{*}\right)\end{array}$ \\
\hline IV & $\begin{array}{l}\text { pump-and- } \\
\text { treat }\end{array}$ & A-E & $4 \& 5$ & b & $\begin{array}{l}(52)-(54), \\
(56),(57)\end{array}$ \\
\hline
\end{tabular}

* capture constraint is specified by initial plume configuration

Table 3

Problem Domains

\begin{tabular}{|l|l|l|l|l|}
\hline Domain & Description & $\mu_{\log K}$ & $\sigma_{\log K}^{2}$ & $\eta_{x}, \eta_{y}, \eta_{z}$ \\
\hline \hline A & homogeneous & -4.3 & NA & NA \\
\hline B & layered & -4.3 & 1 & NA \\
\hline C & random field I & -4.3 & 1 & $5,5,0.75$ \\
\hline D & random field II & -4.3 & 2 & $5,5,0.75$ \\
\hline E & random field III & -4.3 & 1 & $50,50,7.5$ \\
\hline
\end{tabular}

Note: $\log$ refers to $\log _{10}$

\subsection{Domains}

Five domains are summarized in Table 3 according to a general description and properties of a scalar $K$, which distinguishes these domains. The spatial domain for all cases is $\Omega \in[0,1000] \times[0,1000] \times[0,30] \mathrm{m}$.

The medium properties are homogeneous for Domain A, with $K=5.01 \times 10^{-5}$ $\mathrm{m} / \mathrm{s}$. For Domain B, the domain consists of 10 layers of equal thickness with $K$ described as uniform in each layer, log-normally distributed, and uncorrelated

in space. The $K$ distribution for Domain B can be downloaded by following the instructions given at the end of the web page. For Domains $\mathrm{C}-\mathrm{E}, K$ is a correlated random field following a normal distribution in log-transformed space where $\mu_{\log K}$ and $\sigma_{\log K}^{2}$ are the mean and variance of $K$ in $\log _{10}$ space. $K$ is spatially correlated according to the exponential covariance model

$$
\operatorname{cov}(\nu)=\sigma_{\log K}^{2} \exp \left(-\sqrt{\frac{\nu_{x}^{2}}{\eta_{x}^{2}}+\frac{\nu_{y}^{2}}{\eta_{y}^{2}}+\frac{\nu_{z}^{2}}{\eta_{z}^{2}}}\right)
$$


where $\nu, \nu_{x}, \nu_{y}$, and $\nu_{z}$ are spatial coordinates and $\eta_{x}, \eta_{y}$, and $\eta_{z}$ are spatial correlation scales in the $x, y$, and $z$ directions. Domains $\mathrm{C}$ and $\mathrm{D}$ differ from each other in the value of $\log \mathrm{K}$ variance, $\sigma_{\log K}^{2}$. Domains $\mathrm{C}$ and $\mathrm{E}$ differ from each other in the value of the correlation scales $\eta_{x}, \eta_{y}$, and $\eta_{z}$.

\subsection{Mathematical Models}

A variety of mathematical models with varying degrees of sophistication may be applied to approximate the applications outlined in Table 2. Table 4 summarizes the mathematical models detailed below. We specify the models in mathematical form only, so as not to preclude a given simulator, which might stifle advancement of the development of improved simulators. We anticipate comparing among solution methods that share a common set of simulators along with methods with different simulators.

Model 1 follows eqn (9), as applied to confined single-phase flow. The auxiliary conditions for Model 1 are

$$
\begin{aligned}
\left.\frac{\partial h}{\partial x}\right|_{x=0}=\left.\frac{\partial h}{\partial y}\right|_{y=0} & =\left.\frac{\partial h}{\partial z}\right|_{z=0}=0, t>0 \\
q_{z}(x, y, z=30, t>0) & =-1.903 \times 10^{-8}(\mathrm{~m} / \mathrm{s}) \\
h(x=1000, y, z, t>0) & =50-0.001 y(\mathrm{~m}) \\
h(x, y=1000, z, t>0) & =50-0.001 x(\mathrm{~m}) \\
\mathcal{S}(x, y, z, t>0) & =0.0 \\
h(x, y, z, t=0) & =h_{s}
\end{aligned}
$$

where $q_{z}$ is the Darcy flux out of the domain, $h_{s}$ represents the steady-state solution to the flow problem in the absence of additional sources and sinks provided by a specified design, and $S_{s}=10^{-6}(1 / \mathrm{m})$.

Model 2 is similar to Model 1 but applies eqn (9) to an unconfined flow system with the auxiliary conditions given as

$$
\begin{aligned}
\left.\frac{\partial h}{\partial x}\right|_{x=0}=\left.\frac{\partial h}{\partial y}\right|_{y=0} & =\left.\frac{\partial h}{\partial z}\right|_{z=0}=0, t>0 \\
q_{z}(x, y, z=h, t>0) & =-1.903 \times 10^{-8}(\mathrm{~m} / \mathrm{s}) \\
h(x=1000, y, z, t>0) & =20-0.001 y(\mathrm{~m}) \\
h(x, y=1000, z, t>0) & =20-0.001 x(\mathrm{~m}) \\
\mathcal{S}(x, y, z, t>0) & =0.0 \\
h(x, y, z, t=0) & =h_{s}
\end{aligned}
$$


where $S_{s}=2 \times 10^{-1}(1 / \mathrm{m})$, representing the specific yield of the unconfined aquifer. In Models 1 and 2, it is assumed that water is released from storage instantly with a change in head.

Model 3 is appropriate for simulating seawater intrusion, and involves unconfined flow, eqn (9), and conservative species transport, eqn (10). Density dependence is considered via a concentration-dependent expression

$$
\rho=\rho_{0}+\frac{\partial \rho}{\partial C^{\iota}}\left(C^{\iota}-C_{b}^{\iota}\right)
$$

where $\rho_{0}$ is the density of freshwater at the reference temperature, which is assumed to be time and space invariant, $\partial \rho / \partial C^{\iota}$ is the change in density with respect to solute concentration, and $C_{b}^{\iota}$ is the background concentration of solute in the freshwater, taken as $C_{b}^{\iota} \approx 0$. The specific storage in eqn (9) is adjusted for changes in density by a linear approximation: $S_{s}=\left[2 \times 10^{-1}\left(\rho / \rho_{0}\right)\right]$ $(1 / \mathrm{m})$.

The auxiliary conditions for Model 3 are

$$
\begin{aligned}
\left.\frac{\partial h}{\partial x}\right|_{x=0}=\left.\frac{\partial h}{\partial x}\right|_{x=1000} & =\left.\frac{\partial h}{\partial z}\right|_{z=0}=0, t>0 \\
p(x, y=0, z, t>0) & =\rho_{s w} g z \\
q_{y}(x, y=1000, z, t>0) & =-1.282 \times 10^{-5}(\mathrm{~m} / \mathrm{s}) \\
h(x, y, z, t=0) & =h_{s} \\
\left.\frac{\partial C^{\iota}}{\partial x}\right|_{x=0}=\left.\frac{\partial C^{\iota}}{\partial x}\right|_{x=1000} & =\left.\frac{\partial C^{\iota}}{\partial z}\right|_{z=h}=0, t>0 \\
C^{\iota}(x, y=0, z, t>0) & =C^{s w}, t>0 \\
C^{\iota}(x, y=1000, z, t>0) & =0, t>0 \\
C^{\iota}(x, y, z, t=0) & =C_{0}^{\iota}(x, y, z)
\end{aligned}
$$

where $\rho_{s w}$ is the density of seawater, $C_{s w}^{\iota}$ is the concentration of solute in seawater $\left(C_{s w}^{\iota}=35 \mathrm{~kg} / \mathrm{m}^{3}\right)$, and $C_{0}^{\iota}$ represents the steady-state solution to the solute transport problem in the absence of additional sources and sinks provided by a specified design.

Models 4 and 5 are appropriate for species transport as described by eqn (10) and the auxiliary conditions given as

$$
\begin{aligned}
\left.\frac{\partial C^{\iota}}{\partial x}\right|_{x=0}=\left.\frac{\partial C^{\iota}}{\partial y}\right|_{y=0} & =\left.\frac{\partial C^{\iota}}{\partial z}\right|_{z=0}=\left.\frac{\partial C^{\iota}}{\partial z}\right|_{z=h}=0, t>0 \\
C^{\iota}(x=1000, y, z, t>0) & =C^{\iota}(x, y=1000, z, t>0)=0 \\
C^{\iota}(x, y, z, t=0) & =C_{0}^{\iota}(x, y, z)
\end{aligned}
$$


where $C_{0}^{\iota}(x, y, z)$ is the initial contaminant distribution. The unperturbed (no pumping) flow fields for Models 4 and 5 are provided by the unconfined flow Model 2 applied to a choice from the set of possible Domains A-E. Applications III and IV require initial contaminant plumes that are expected to be captured or remediated. The initial concentration field, $C_{0}^{\iota}$, may be generated by simulating plume development from a finite source for $t \in\left[-t_{s}, 0\right]$; for additional details see the SI.

In Model 4, a single, conservative species $(\iota=c)$ is considered, such that $\mathcal{I}^{c}=0$ and $\mathcal{R}^{c}=0$. This model is used to generate the plume to be captured in Application III and remediated in Application IV.

In Model 5, a conservative $(\iota=c)$ and a sorbing $(\iota=r)$ species are considered, $C^{c}$ and $C^{r}$, respectively. Model 5 is to be used with Application IV. For the sorbing species, aqueous-solid phase mass exchange is described by a first-order rate model

$$
\mathcal{I}^{r}=\theta^{s} \rho^{s} k_{f}\left(\omega^{r s e}-\omega^{r s}\right)
$$

where the Freundlich equilibrium model describes the relationship between the solid phase mass fraction and the fluid phase concentration at equilibrium

$$
\omega^{r s e}=K_{f}\left(C^{r}\right)^{n_{f}}
$$

where $r$ is a species qualifier for the sorbing species, $k_{f}$ is a first-order mass transfer rate coefficient, $\omega^{r s e}$ is the solid-phase mass fraction in equilibrium with the fluid-phase concentration, $\omega^{r s}$ is the solid-phase mass fraction, $K_{f}$ is a Freundlich capacity coefficient, and $n_{f}$ is an exponent related to the sorption energy. A separate mass balance equation for the mass fraction of the species sorbed to the solid phase must be solved,

$$
\frac{\partial \omega^{r s}}{\partial t}=k_{f}\left(\omega^{r s e}-\omega^{r s}\right)
$$

in addition to solving the solute species mass balances.

\subsection{Objective Functions}

The objective function for each application is indicated in Table 2 and includes a capital cost $f^{c}$ and an operational cost $f^{o}$, which depends upon time. The generic objective is to minimize the sum of $f^{c}+f^{o}$. The objective function for all applications and models depends upon the number, 
Table 4

Model Formulations

\begin{tabular}{|l|l|l|}
\hline Model & Description & $\begin{array}{l}\text { Model Equa- } \\
\text { tions }\end{array}$ \\
\hline \hline 1 & confined flow & $(9),(21)-(26)$ \\
\hline 2 & unconfined flow & $(9),(27)-(32)$ \\
\hline 3 & $\begin{array}{l}\text { unconfined, density-dependent flow/conservative } \\
\text { species transport }\end{array}$ & $\begin{array}{l}(9)-(11),(27)- \\
(41)\end{array}$ \\
\hline 4 & unconfined flow/conservative species transport & $\begin{array}{l}(9)-(11),(27)- \\
(32),(42)-(44)\end{array}$ \\
\hline 5 & $\begin{array}{l}\text { unconfined flow/conservative and retarded species } \\
\text { transport }\end{array}$ & $\begin{array}{l}(9)-(11),(27)- \\
(32),(42)-(47)\end{array}$ \\
\hline
\end{tabular}

location, and extraction/injection rate of all wells. This amounts to specification of $Q_{i}\left(\mathbf{x}_{i}, t\right), i=1, \ldots, n$. The set of $\{\mathbf{Q}\}$ are ordered such that the extraction rates are $Q_{i}<0, i=1, \ldots, n^{e}$ and the injection rates are $Q_{i}>0, i=n^{e}+1, \ldots, n$, where $n^{e}$ is the number of extraction wells, and the number of injection wells is $n^{i}=n-n^{e}$.

The capital cost for a well includes the well construction and, for the case of an extraction well, the cost of a pump. Injection wells are assumed to discharge under gravity feed conditions. The functional form for the capital cost is

$$
f_{a}^{c}=\sum_{i=1}^{n} c_{0} d_{i}^{b_{0}}+\sum_{i=1}^{n^{e}} c_{1}\left|Q_{i}^{m}\right|^{b_{1}}\left(z_{g s}-h^{m i n}\right)^{b_{2}}
$$

and the operational cost is

$$
f_{a}^{o}=\int_{0}^{t_{f}}\left(\sum_{i=1}^{n^{e}} c_{2} Q_{i}\left(h_{i}-z_{g s}\right)+\sum_{i=n^{e}+1}^{n} c_{3} Q_{i}\right) \mathrm{d} t
$$

where $b_{j}$ and $c_{j}$ are coefficients and exponents of the cost model, respectively; $d_{i}$ is the depth of the well below ground surface, $Q_{i}^{m}$ is the design pumping rate, $z_{g s}$ is the ground surface elevation, $h^{\text {min }}$ is the minimum allowable head, $t_{f}$ is the total time of operation, $h_{i}$ is the hydraulic head in each well.

For the PAT application, the objective function includes not only the well construction and operation costs but also capital and operational costs related to treatment. We base our treatment cost functions on a cost model for air stripping resulting in the capital cost function

$$
f_{b}^{c}=f_{a}^{c}+c_{4} Z
$$


Table 5

Objective Functions

\begin{tabular}{|l|l|l|}
\hline$f$ forms & Description & Equations \\
\hline \hline $\mathrm{a}$ & installation + extraction + injection & $\begin{array}{l}f_{a}=f_{a}^{c}+f_{a}^{o} \\
(48),(49)\end{array}$ \\
\hline $\mathrm{b}$ & installation + extraction + injection + treatment & $\begin{array}{l}f_{b}=f_{b}^{c}+f_{b}^{o} \\
(50),(51)\end{array}$ \\
\hline
\end{tabular}

and the operational cost function

$$
f_{b}^{o}=f_{a}^{o}+\int_{0}^{t_{f}} Z\left(c_{5}-c_{6} Q_{T}^{e}\right) \mathrm{d} t
$$

where $Z$ is the total height of the stripping tower required, and $Q_{T}^{e}=\sum_{i=1}^{n^{e}} Q_{i}$ is the total extraction rate.

\subsection{Constraints}

The objective functions listed in Table 5 are constrained by hydraulic and solute mass constraints. For Applications I and II there are constraints on the total extraction rate due to the well-field demand, the extraction and injection rate at each well, and the aquifer drawdown

$$
\begin{gathered}
Q_{T} \leq Q_{T}^{\min } \\
Q^{e \max } \leq Q_{i} \leq Q^{i m a x}, i=1, \ldots, n \\
h^{\max } \geq h_{i} \geq h^{\text {min }} i=1, \ldots, n
\end{gathered}
$$

where $Q_{T}=\sum_{i=1}^{n} Q_{i}$ is the net pumping rate, $Q_{T}^{m i n}$ is the minimum total extraction rate, $Q^{e m a x}$ is the maximum extraction rate at any well, $Q^{\text {imax }}$ is the maximum injection rate at any well, $h^{\max }$ is the maximum allowable head, and $h^{\text {min }}$ is the minimum allowable head.

For Application II an additional constraint is specified for the maximum solute concentration in the extraction wells

$$
\frac{C_{i}^{\iota}}{C_{s w}^{\iota}} \leq F_{s w} \quad i=1, \ldots, n^{e}
$$

where $C_{i}^{\iota}$ is the solute concentration at extraction well $i$, and $F_{s w}$ is the maximum allowable normalized solute concentration. 
For Application III, we also constrain the total net extraction rate

$$
\sum_{i=1}^{n} Q_{i} \geq Q_{T}^{\max }
$$

where $Q_{T}^{\max }$ is the maximum total extraction rate. The plume capture constraint is specified by the boundary of the initial contaminant distribution for each model domain. We leave it to the modeler to choose the physical and mathematical representation of the constraint (e.g. advective control vs. gradient control).

Application IV requires a constraint specifying the aquifer cleanup performance. To provide a common metric, we constrain the maximum global fraction of mass that may remain in the system at a time $t_{f}$, which is possible to do for the numerical studies of interest here but difficult to measure in practice. The fractional mass remaining constraint is

$$
\frac{\int_{\Omega}\left(\theta^{a} C^{\iota}+\theta^{s} \rho^{s} \omega^{\iota s}\right)_{t=t_{f}} \mathrm{~d} \Omega}{\int_{\Omega}\left(\theta^{a} C^{\iota}+\theta^{s} \rho^{s} \omega^{\iota s}\right)_{t=0} \mathrm{~d} \Omega} \leq M
$$

where $M$ is the maximum fractional mass remaining at time $t_{f}$. When $\iota=c$ the solute mass on the solid-phase contribution is identically zero. However, when $\iota=r$ sorption to the solid phase must be accounted for when computing the fractional mass remaining in the system. In practice, remediation constraints are often based upon concentrations, but such simulated values are affected by many factors, such as numerical discretization details, screened intervals of a well, etc., that we wish to avoid.

\subsection{Comparative Principles}

Comparison among competing solution methods for optimal design is not trivial. In general, two types of criteria are of interest: (1) the objective function value achieved at the termination of the optimization and (2) the computational effort expended. Care is necessary in achieving and documenting with scientific certainty both of these criteria. One problem when comparing among different methods is that, since the termination criteria varies from method to method or even from user to user, comparisons of objective function values and computational effort are not straightforward.

We suggest the following principles as general guidance to facilitate comparisons among competing methods: 
(1) computational algorithms should be reported in sufficient detail to reproduce the results directly or with reference to a peer-reviewed publication;

(2) the computational platform should be fully specified including make, model, processor type and speed; RAM, operating system and release; language(s) used; compiler make, release, and switches; and complete details of any third-party or public domain software used including release details;

(3) objective function values should be confirmed by accurate, grid-independent numerical solution of the specified problem;

(4) non-deterministic solution methods should include a measure of the error in the objective function value returned;

(5) claims of a global minimum solution should be supported by results from multiple approaches with multiple starting locations, and where feasible with a grid search - a more realistic goal is a significant improvement in the best previously obtained optimal solution for a given problem;

(6) optimization results depending upon free parameters should report on the values of those parameters, the sensitivity of results to those parameters, and ideally on methods to estimate good values of all free parameters;

(7) computational effort should be reported in terms of the number, CPU time, and operations of all types and levels of function evaluations and approximate evaluations; the optimization algorithm; and all other significant uses of CPU time;

(8) the scalability of the optimization with regard to the number of decision variables, i.e., the relationship of $\mathrm{CPU}$ time to the number of decision variables should be investigated and reported; and

(9) if a parallel solution approach is used, scalability of the results in terms of total CPU time required as a function of the number of processors used in the solution should be reported.

\subsection{Summary}

The combinations of models, objective functions, constraints, and model domains described in the previous sections will pose many of the difficulties anticipated in solving real-world problems:

(1) The objective functions and constraints include integer terms that depend on the decision variables and on nonlinear relationships between the constraints and the decision variables.

(2) Evaluation of the objective function is based on solving model equations, especially the transport equation, that are difficult to solve accurately and quickly.

(3) The combination of flow boundary conditions and hydraulic conductivity distributions gives rise to extremely complex relationships between the 
objective function, the decision variables, the constraints, and the state variables.

(4) The number and range of decision variables is potentially enormous, requiring either very fast solutions or judicious narrowing of the decision space.

There are several directions in which these test problems can be extended. In general, we advocate the continued evolution of community test problems that are increasingly more realistic in terms of our representation of the physical and chemical characteristics of the subsurface environment, contaminant sources, and remediation approaches. We also advocate coupling data collection for stochastic systems with assessments of the value of information, considering multi-objective problems, and implementing strategies with recourse.

\section{CONCLUSIONS}

We have reviewed critically the literature in the subsurface hydrology field related to optimal design. While significant work has been done, much remains to be done before a mature level of understanding is achieved. Based upon our review of the literature we draw several conclusions: (1) optimization problems of central importance are nonlinear and usually have multiple local minima; (2) models used to represent subsurface systems are usually stochastic in nature and expensive to solve, especially when highly resolved; (3) optimal decisions often involve multiple, competing objective functions; (4) conventional approaches used to solve optimal subsurface design problems often lack efficiency and robustness; and (5) in-depth comparisons among methods are lacking.

We have suggested several new approaches for solving subsurface optimization problems. We describe a class of optimization methods, deterministic sampling methods, that have only recently been used in the subsurface optimization community, but are promising with regard to general properties and encouraging results that have been obtained in many other challenging scientific fields. We describe alternative approaches for solving stochastic optimization problems, such as techniques for assessing cross-covariances between uncertain parameters and state variables, stochastic finite element methods, second order cone optimization, and Bayesian maximum entropy methods. Finally, we suggest a range of approaches to apply to multi-objective optimization problems, with a focus on incorporating maximizing design reliability as an objective and utilizing decision analysis techniques to determine preferences among multiple objectives. We strongly advocate the adoption of both stochastic and multi-objective frameworks for the optimal design of subsurface flow and transport systems. 
We end the paper with a detailed set of optimization problems for the community to consider for benchmarking among the various optimization approaches. The problems consider the following design applications: well field design, well field design under the influence of seawater intrusion, hydraulic capture of a contaminant plume, and pump-and-treat remediation of a two-species contaminant plume. The applications are specified in terms of the decision variables, the physical domains, the governing flow and transport problems to be solved, the objective functions, and the constraints on state and decision variables. We consider the size and complexity of the problems to be more realistic than those typically considered to date.

\section{Acknowledgements}

The efforts of ASM were supported by NSF grant BES-0083112 and USEPA grant CR826614-01-0. Part of ASM's efforts took place while on sabbatical at the Technical University of Delft, The Netherlands, where he was supported by a Fulbright Scholarship and TUDelft Visiting Scholarship. The efforts of CTK were supported by NSF grants DMS-0070641 and DMS-0112542, and ARO grant DAAD19-99-1-0186. The efforts of CTM were supported by the National Institute of Environmental Health Sciences grant 5 P42 ES05948-02 and National Science Foundation grant DMS-0112653. Computational support was provided by the North Carolina Supercomputer Center to CTK and CTM. 


\section{References}

[1] U.S. Environmental Protection Agency. www.cluin.org. Technology Innovation Office, Washington, DC, 2001.

[2] D. Ahlfeld and A. Mulligan. Optimal management of flow in groundwater systems. Academic Press, Orlando, FL, 1999.

[3] D. P. Ahlfeld and E. H. Hill III. The sensitivity of remedial strategies to design criteria. Ground Water, 34(2):341-348, 1996.

[4] D. P. Ahlfeld, J. M. Mulvey, and G. F. Pinder. Designing optimal strategies for contaminated groundwater remediation. Advances in Water Resources, 9:77-84, 1986.

[5] D. P. Ahlfeld, J. M. Mulvey, and G. F. Pinder. Combining physical containment with optimal withdrawal for contaminated groundwater remediation. Advances in Water Resources, 10:200-204, 1987.

[6] D. P. Ahlfeld, J. M. Mulvey, and G. F. Pinder. Contaminated groundwater remediation design using simulation, optimization, and sensitivity theory 2. Analysis of a field site. Water Resources Research, 24(3):443$452,1988$.

[7] D. P. Ahlfeld, J. M. Mulvey, G. F. Pinder, and E. F. Wood. Contaminated groundwater remediation design using simulation, optimization, and sensitivity theory 1. model development. Water Resources Research, 24(3):431-441, 1988.

[8] D. P. Ahlfeld, R. H. Page, and G. F. Pinder. Optimal ground-water remediation methods applied to a superfund site: From formulation to implementation. Ground Water, 33(1):58-70, 1995.

[9] D. P. Ahlfeld and M. P. Sprong. Presence of nonconvexity in groundwater concentration response functions. Journal of Water Resources Planning and Management, 124(1):8-14, 1998.

[10] D. P. Ahlfeld, A. Zafirakou, and R. G. Riefler. Solution of the groundwater transport managment problem by sequential relaxation. Advances in Water Resources, 21(7):591-604, 1998.

[11] D.P. Ahlfeld and M.P. Sprong. Presence of non-convexity in the groundwater concentration response function. ASCE, Journal of Water Resources Planning and Management, 124(1):8-14, 1998.

[12] A. H. Aly and R. C. Peralta. Comparison of a genetic algorithm and mathematical programming to the design of groundwater cleanup systems. Water Resources Research, 35(8):2415-2425, 1999.

[13] A. H. Aly and R. C. Peralta. Optimal design of aquifer cleanup systems under uncertainty using a neural network and a genetic algorithm. Water Resources Research, 35(8):2523-2532, 1999.

[14] R. Andricevic. A real-time approach to management and monitoring of groundwater hydraulics. Water Resources Research, 26(11):2747-2755, 1990.

[15] C. Audet and J. E. Dennis. On the convergence of pattern search algorithms with mixed variables. Technical Report TR99-02, Department 
of Computational and Applied Mathematics, Rice University, Houston Texas, 1998. to appear in SIAM J. on Optimization.

[16] Charles Audet, Andrew J. Booker, Paul D. Frank, and Douglas W. Moore. A surrogate-model-based method for constrained optimization. In AIAA/USAF/NASA/ISSMO Symposium on Multidisciplinary Analysis and Optimization, volume AIAA no.4891. AIAA, 2000.

[17] Charles Audet and J. E. Dennis. Pattern search algorithms for mixed variable programming. SIAM J. Optim., 11:573-594, 2000.

[18] Charles Audet and J. E. Dennis, Jr. Analysis of generalized pattern searches. (http://www. caam.rice.edu/caam/trs/2000/TR00-07.ps), 2000.

[19] C. A. Baker, L. T. Watson, B. Grossman, R. T. Haftka, and W. H. Mason. Parallel global aircraft configuration design space exploration. preprint, 1999.

[20] C. A. Baker, L. T. Watson, B. Grossman, W. H. Mason, S. E. Cox, and R. T. Haftka. Study of a global design space exploration method for aerospace vehicles. preprint, 1999.

[21] A. Battermann, J. M. Gablonsky, A. Patrick, C. T. Kelley, T. Coffey, K. Kavanagh, and C. T. Miller. Solution of a groundwater control problem with implicit filtering. Technical Report CRSC-TR00-30, North Carolina State University, Center for Research in Scientific Computation, December 2000.

[22] J. Bear. Hydraulics of Groundwater. McGraw-Hill, New York, 1979.

[23] J. Bear and Y. Sun. Optimization of pump-treat-inject (PTI) design for the remediation of a contaminated aquifer: Multi-stage design with chance constraints. Journal of Contaminant Hydrology, 29:225-244, 1998.

[24] A. Ben-Tal and A. Nemirovski. Convex optimization in engineering. Technical report, Technion-Israel Institute of Technlogy, Haifa, Israel, 1998.

[25] J. T. Betts. Practical Methods for Nonlinear Control Using Nonlinear Programming. Number 3 in Advances in Design and Control. SIAM, Philadelphia, 2000.

[26] P. Bogaert, M. L. Serre, and G. Christakos. Efficient computational BME analysis of non-Gaussian data in terms of transformation functions. In Proceedings of the Fifth Annual Conference of the International Association for Mathematical Geology, volume I, pages 57-62, 1999.

[27] A. J. Booker, J. E. Dennis, P. D. Frank, D. B. Serafini, V. Torczon, and M. W. Trosset. A rigorous framework for optimization of expensive function by surrogates. Structural Optimization, 17:1-13, 1999.

[28] R. Carter, J. M. Gablonsky, A. Patrick, C. T. Kelley, and O. J. Eslinger. Algorithms for noisy problems in gas transmission pipeline optimization. Optimization and Engineering, 2:139-157, 2001.

[29] R. G. Carter. Nonsequential dynamic programming for optimizing pipelines, 1999. Presentation at the 1999 SIAM Conference on Opti- 
mization.

[30] R. G. Carter, W. W. Schroeder, and T. D. Harbick. Some causes and effect of discontinuities in modeling and optimizing gas transmission networks, 1993. Proceedings of the Pipeline Simulation Interest Group, Pittsburg PA, Paper number PSIG-9308.

[31] L. C. Chang, C. A. Shoemaker, and P. L. F. Liu. Optimal timevarying pumping rates for groundwater remediation - application of a constrained optimal control algorithm. Water Resources Research, 28 (12):3157-3173, 1992.

[32] T. D. Choi. Bound Constrained Optimization. PhD thesis, North Carolina State University, Raleigh, North Carolina, 1999.

[33] T. D. Choi, O. J. Eslinger, P. Gilmore, A. Patrick, C. T. Kelley, and J. M. Gablonsky. IFFCO: Implicit Filtering for Constrained Optimization, Version 2. Technical Report CRSC-TR99-23, North Carolina State University, Center for Research in Scientific Computation, July 1999.

[34] T. D. Choi, O. J. Eslinger, C. T. Kelley, J. W. David, and M. Etheridge. Optimization of automotive valve train components with implicit filtering. Optimization and Engineering, 1:9-28, 2000.

[35] T. D. Choi and C. T. Kelley. Superlinear convergence and implicit filtering. SIAM J. Optim., 10:1149-1162, 2000.

[36] G. Christakos. Random Field Models in the Earth Sciences. Academic Press, San Diego, CA, 1992.

[37] G. Christakos, D. T. Hristopulos, and C. T. Miller. Stochastic diagrammatic analysis of groundwater flow in heterogeneous porous media. Water Resources Research, 31(7):1687-1703, 1995.

[38] G. Christakos, C. T. Miller, and D. Oliver. The development of stochastic space transformation and diagrammatic perturbation techniques in subsurface hydrology. Stochastic Hydrology and Hydraulics, 7(1):14-32, 1993.

[39] G. Christakos, C. T. Miller, and D. Oliver. Stochastic flow modelling in terms of interactive perturbation, Feynman diagrams and graph theory. IEEE, Banking on Technology, BT-93:77-84, 1993.

[40] C.A. Coello Coello. A short tutorial on evolutionarymultiobjective optimization. In Evolutionary Multi-Criterion Optimization, E. Zitzler et al., eds., pages 21-40. Lecture Notes in Computer Science, Springer, 2001.

[41] C.A. Coello Coello and G.T. Pulido. A micro-genetic agorithm for multiobjective optimization. In Evolutionary Multi-Criterion Optimization, E. Zitzler et al., eds., pages 126-140. Lecture Notes in Computer Science, Springer, 2001.

[42] A. R. Conn, K. Scheinberg, and Ph. L. Toint. A derivative free optimization algorithm in practice. Proceeedings of 7-th AIAA/USAF/NASA/ISSMO Symposium on Multidisciplinary Analysis and Optimization, St Louis, MO. Sept 2-4, 1998., 1998.

[43] T. B. Culver and C. A. Shoemaker. Dynamic optimal control for ground- 
water remediation with flexible management periods. Water Resources Research, 28(3):629-641, 1992.

[44] T. B. Culver and C. A. Shoemaker. Differentiating a finite element biodegradation simulation model for optimal control. Water Res. Research, 32:187-192, 1996.

[45] T. B. Culver and C. A. Shoemaker. Dynamic optimal ground-water reclamation with treatment capital costs. Journal of Water Resources Planning and Management, 123(1):23-29, 1997.

[46] T. B. Culver and C. A. Shoemaker. Dynamic optimal ground-water reclamation with treatment capital costs. Journal of Water Resources Planning and Management, 123:23-29, 1997.

[47] T.B. Culver and G.W. Shenk. Dynamic optimal groundwater remediation by granular activated carbon. ASCE, Journal of Water Resources Planning and Management, 124(1):59-64, 1998.

[48] G. Dagan. Flow and Transport in Porous Media. Springer-Verlag, New York, 1989.

[49] K. Deb, S Agrawal, A. Pratap, and T. Meyarivan. A fast elitist nondominated sorting genetic algorithm for multi-objective optimization: Nsga-ii. In Proceedings of the Parallel Problem Solving from Nature VI Conference, pages 849-858. Lawrence Erlbaum, 2000.

[50] L. Duckstein, Goicoechea, and D. Hansen. Multiobjective system analysis with engineering and Business application. Wiley, New York, 1982.

[51] M. Erickson, A. Mayer, and J. Horn. Multi-objective optimal design of groundwater remediation systems: Application of the niched pareto genetic algorithm (npga). Advances in Water Resources, 25:51-65, 2002.

[52] M. Etheridge. Preliminary performance of carbon-carbon valves in high speed pushrod type valve trains. Master's thesis, North Carolina State University, Raleigh, North Carolina, 1998.

[53] C.M. Fonseca and P.J Fleming. Multiobjective optimization and multiple constraint handling with evolutionary algorithms i: A unified formulation. IEEE Transactions on Systems, Man, and Cybernetics, Part A: Systems and Human, 28(1):26-37, 1998.

[54] S. J. Fortune, D. M. Gay, B. W. Kernighan, O. Landron, R. A. Valenzuela, and M. H. Wright. WISE design of indoor wireless systems. IEEE Computational Science and Engineering, 2:58-68, 1995.

[55] R. A. Freeze. A stochastic-conceptual analysis of one-dimensional groundwater flow in nonuniform homogeneous media. Water Resources Research, 11(5):725-741, 1975.

[56] R. A. Freeze and S. M. Gorelick. Convergence of stochastic optimization and decision analysis in the engineering design of aquifer remediation. Ground Water, 37(6):934-954, 1999.

[57] R. A. Freeze, B. James, J. Massmann, T. Sperling, and L. Smith. Hydrogeological decision analysis .4. the concept of data worth and its use in the development of site investigation strategies. Ground Water, 30 (4):574-588, 1992. 
[58] R. A. Freeze, J. Massmann, L. Smith, T. Sperling, and B. James. Hydrogeological decision analysis: 1. a framework. Ground Water, 28(5): 738-766, 1990.

[59] J. Gablonsky. An implementation of the DIRECT algorithm. Technical Report CRSC-TR98-29, North Carolina State University, Center for Research in Scientific Computation, August 1998.

[60] J. M. Gablonsky and C. T. Kelley. A locally-biased form of the DIRECT algorithm. Journal of Global Optimization, 21:27-37, 2001.

[61] L. W. Gelhar. Stochastic Subsurface Hydrology. Prentice Hall, Englewood Cliffs, NJ, 1993.

[62] A.M. Geoffrion, J.S. Dyer, and A. Feinberg. An interactive approach for multi-criterion optimization, with an application to the operation of an academic department. Management Science, 19(4):357-368, 1972.

[63] R. Ghanem. Probabilistic characterization of transport in heterogeneous media. Computer Methods in Applied Mechanics and Engineering, 158: 199-220, 1998.

[64] R. Ghanem. Scales of fluctuation and the propagation of uncertainty in random porous media. Water Resources Research, 34(9):2123-2136, 1998.

[65] R. Ghanem and S. Dham. Stochastic finite element analysis for multiphase flow in heterogeneous porous media. Transport in Porous Media, 32:239-262, 1998.

[66] A. Gharbi and R. C. Peralta. Integrated embedding optimization applied to Salt Lake valley aquifers. Water Resources Research, 30(3):817-832, 1994.

[67] P. Gilmore and C. T. Kelley. An implicit filtering algorithm for optimization of functions with many local minima. SIAM J. Optim., 5: 269-285, 1995.

[68] P. A. Gilmore, S. S. Berger, R. F. Burr, and J. A. Burns. Automated optimization techniques for phase change piezoelectric ink jet performance enhancement. In 1997 International Conference on Digital Printing Technologies, pages 716-721. Society for Imaging Science and Technology, IS\&T's NIP 13, November, 1997.

[69] D.E. Goldberg. Genetic algorithms. Addison-Wesley, Reading, MA, 1989.

[70] S. M. Gorelick and I. Remson. Optimal dynamic management of groundwater pollutant sources. Water Resources Research, 18(1):71-76, 1982.

[71] S. M. Gorelick, C. I. Voss, P. E. Gill, W. Murray, M. A. Saunders, and M. H. Wright. Aquifer reclamation design: The use of contaminant transport simulation combined with nonlinear programming. Water Resources Research, 20(4):415-427, 1984.

[72] S.M. Gorelick, R.A. Freeze, D. Donohue, and J.F. Keely. Groundwater Contamination - Optimal Capture and Containment. Lewis Publishers, Boca Raton, Florida, 2001.

[73] A. Griewank. Evaluating Derivatives: Principles and Techniques of Algo- 
rithmic Differentiation. Number 19 in Frontiers in Applied Mathematics. SIAM, Philadelphia, 2000.

[74] R. Haggerty and S. M. Gorelick. Design of multiple contaminant remediation: Sensitivity to rate-limited mass transfer. Water Resources Research, 30(2):435-446, 1994.

[75] H.H. Herke, U. Hornung, Y. Kelanemer, M. Slodicka, and S. Schumacher. Optimal control of soil venting: Mathematical modeling and applications. Birkhauser, Basel, Switzerland, 1999.

[76] A. B. C. Hilton and T. B. Culver. Constraint handling for genetic algorithms in optimal remediation design. Journal of Water Resources Planning and Management, 126(3), 2000.

[77] R. Hooke and T. A. Jeeves. 'Direct search' solution of numerical and statistical problems. Journal of the Association for Computing Machinery, 8:212-229, 1961.

[78] J. Horn. The nature of niching: Genetic algorithms and the evolution of optimal, cooperative populations. PhD thesis, University of Illinois at Urbana Champaign, Urbana Champaign, IL, 1997.

[79] C. Huang and A. S. Mayer. Pump-and-treat optimization using well locations and pumping rates as decision variables. Water Resources Research, 33(5):1001-1012, 1997.

[80] B. R. James and S. M. Gorelick. When enough is enough: The worth of monitoring data in aquifer remediation design. Water Resources Research, 30(12):3499-3513, 1994.

[81] D. R. Jones, C. C. Perttunen, and B. E. Stuckman. Lipschitzian optimization without the Lipschitz constant. J. Optim. Theory Appl., 79: 157-181, 1993.

[82] G. P. Karatzas and G. F. Pinder. Groundwater management using numerical simulation and the outer approximation method for global optimization. Water Resources Research, 29(10):3371-3378, 1993.

[83] G. P. Karatzas and G. F. Pinder. The solution of groundwater quality management problems with a nonconvex feasible region using a cutting plane optimization technique. Water Resources Research, 32(4):10911100, 1996.

[84] A. Keidser and D. Rosbjerg. A comparison of four inverse approaches to groundwater flow and transport parameter identification. Water Resources Research, 27(9):2219-2232, 1991.

[85] C. T. Kelley. Iterative Methods for Optimization. Number 18 in Frontiers in Applied Mathematics. SIAM, Philadelphia, 1999.

[86] Michael Kokkolarus, Charles Audet, and J. E. Dennis. Mixed variable optimization of the number and composition of heat intercepts in a thermal insulation system. Optimization and Engineering, 2:5-30, 2001.

[87] S.-I. Lee and P. K. Kitanidis. Optimal estimation and scheduling in aquifer remediation with incomplete information. Water Resources Research, 27(9):2203-2217, 1991.

[88] R. M. Lewis and V. Torczon. Pattern search algorithms for linearly 
constrained minimization. SIAM J. Optim., 10:917-941, 2000.

[89] M-D. Lin and D.C. McKinney. Optimization of surfactant enhanced pump-and-treat remediation systems. In Proc. ASCE Specialty Conference on Water Resour. Plan. and Mgt., pages 864-867. American Society of Civil Engineers, 1995.

[90] C. M. Mansfield and C. A. Shoemaker. Optimal remediation of unconfined aquifers: Numerical applications and derivative calculations. Water Resources Research, 35(5):1455-1469, 1999.

[91] C. M. Mansfield and C. A. Shoemaker. Optimal remediation of unconfined aquifiers: numerical applications and derivative calculations. Water Res. Research, 35:1455-1469, 1999.

[92] C. M. Mansfield, C. A. Shoemaker, and L.-Z. Liao. Utilizing sparsity in time-varying optimal control of aquifer cleanup. Journal of Water Resources Planning and Management, 124(1):15-21, 1998.

[93] R. A. Marryott, D. E. Dougherty, and R. L. Stollar. Optimal groundwater management .2. application of simulated annealing to a field-scale contamination site. Water Resources Research, 29(4):847-860, 1993.

[94] J. Massmann, R. A. Freeze, L. Smith, T. Sperling, and B. James. Hydrogeological decision analysis: 2 . applications to ground-water contamination. Ground Water, 29(4):536-548, 1991.

[95] A.S. Mayer and C. Huang. The role of uncertainty in the optimization of subsurface systems. In XI International Conference on Computational Methods in Water Resources, pages 635-642. Elsevier, 1996.

[96] D. Q. Mayne. A second-order gradient method for determining optimal trajectories of nonlinear discrete-time systems. Intl. J. Contr., 3:85-95, 1966.

[97] D. C. McKinney, G. B. Gates, and M. D. Lin. Groundwater resource management models: A comparison of genetic algorithms and nonlinear programming. In A. Peters, G. Wittum, B. Herrling, U. Meissner, C. A. Brebbia, W. G. Gray, and G. F. Pinder, editors, Computational Methods in Water Resources X, volume Vol. 2, pages 859-866, Dordrecht, The Netherlands, 1994. Kluwer Academic Publishers.

[98] D. C. Mckinney and M. D. Lin. Approximate mixed-integer nonlinear programming methods for optimal aquifer remediation design. Water Resources Research, 31(3):731-740, 1995.

[99] D.C. McKinney and M.-D. Lin. Approximate mixed-integer nonlinear programming methods for optimal aquifer remediation design. Water Resources Research, 31(3):731-740, 1995.

[100] K. Miettinen and M.M. Makela. Comparative evaluation of some interactive reference-point-based methods for multi-objective optmisation. Journal of the Operational Research Society, 50:949-959, 1999.

[101] C. T. Miller, G. Christakos, P. T. Imhoff, J. F. McBride, J. A. Pedit, and J. A. Trangenstein. Multiphase flow and transport modeling in heterogeneous porous media: Challenges and approaches. Advances in Water Resources, 21(2):77-120, 1998. 
[102] B. S. Minsker and C. A. Shoemaker. Computational issues for optimal in-situ bioremediation design. Journal of Water Resources Planning and Managements, 124(1):39-46, 1998.

[103] B. S. Minsker and C. A. Shoemaker. Quantifying the effects of uncertainty on optimal groundwater bioremediation policies. Water Resources Research, 34(12):3615-3625, 1998.

[104] B. S. Minsker and C. A. Shoemaker. Computational issues for in-situ bioremediation design. Journal of Water Resources Planning and Management, 124:39-46, 1999.

[105] D. R. Morgan, J. W. Eheart, and A. J. Valocchi. Aquifer remediation design under uncertainty using a new chance constrained programming technique. Water Resources Research, 29(3):551-561, 1993.

[106] A. E. Mulligan and D. P. Ahlfeld. Advective control of groundwater contaminant plumes: Model development and comparison to hydraulic control. Water Resources Research, 35(8):2285-2294, 1999.

[107] B. A Murtagh and M. A. Saunders. MINOS 5.1 users guide. Technical Report SOL 83-20R, Dep. of Oper. Res., Stanford Univ., 1987.

[108] J. M. Ndambuki, T. Terlaky, C. B. M. Stroet, and E. J. M. Veiling. Robust groundwater management through second-order cone programming (socp). Technical Report Report \# 2000/11, McMaster University, Hamilton, Ontario, Canada, 2000.

[109] J. A. Nelder and R. Mead. A simplex method for function minimization. Comput. J., 7:308-313, 1965.

[110] S. P. Neumann and G. L. Chen. On instability during immiscible displacement in fractures and porous media. Water Resources Research, 32(6):1891-1894, 1996.

[111] L. D. Oliver and G. Christakos. Diagrammatic solutions for hydraulic head moments in 1-d and 2-d bounded fomains. Stochastic Hydrology and Hydraulics, 9(4):269-296, 1995.

[112] J. A. Pedit and C. T. Miller. Heterogeneous sorption processes in subsurface systems, 1. Model formulations and applications. Environmental Science \& Technology, 28(12):2094-2104, 1994.

[113] R. C. Peralta, M. A. Hegazy, and G. R. Musharrafieh. Preventing pesticide contamination of groundwater while maximizing irrigated crop yield. Water Resources Research, 30(11):3183-3193, 1994.

[114] A. J. Rabideau and C. T. Miller. Two-dimensional modeling of aquifer remediation influenced by sorption nonequilibrium and hydraulic conductivity heterogeneity. Water Resources Research, 30(5):1457-1470, 1994.

[115] P. Reed, B. Minsker, and D. E. Goldberg. Designing a competent simple genetic algorithm for search and optimization. Water Resources Research, 36(12):3757-3761, 2000.

[116] B. J. Ritzel, J. W. Eheart, and S. Ranjithan. Using genetic algorithms to solve a multiple objective groundwater pollution containment problem. Water Resources Research, 30(5):1589-1603, 1994. 
[117] D. M. Rizzo and D. E. Dougherty. Design optimization for multiple management period groundwater remediation. Water Resources Research, 32(8):2549-2561, 1996.

[118] L. L. Rogers and F. U. Dowla. Optimization of groundwater remediation using artificial neural networks with parallel solute transport modeling. Water Resources Research, 30(2):457-481, 1994.

[119] L.L. Rogers and V.M. Johnson. Remediation tradeoffs addressed with simulated annealing optimization. In XII International Conference on Computational Methods in Water Resources, pages 582-590. Elsevier, 1998.

[120] B. Roy. The outranking approach and the foundations of electre method. Theory and Decision Making, 31:49-73, 1991.

[121] K. T. Russell and A. J. Rabideau. Decision analysis for pump-andtreat design. Ground Water Monitoring and Remediation, 20(3):159$168,2000$.

[122] C. S. Sawyer, D. P. Ahlfeld, and A. J. King. Groundwater remediation design using a three-dimensional simulation model and mixed integer programming. Water Resources Research, 31(5):1373-1385, 1995.

[123] S. Sawyer and Y.-F. Lin. Mixed-integer chance-constrained models for ground-water remediation. Journal of Water Resources Planning and Management, 124(5):285-294, 1998.

[124] J. D Schaffer. Multiple objective optimization with vector evaluated genetic algorithms. In Genetic Algorithms and their Applications: Proceedings of the First International Conference on Genetic Algorithms, pages 93-100. Lawrence Erlbaum, 1985.

[125] M. L. Serre and G. Christakos. Modern geostatistics: Computational BME in the light of uncertain physical knowledge - the Equus Beds study. Stochastic Environmental Research and Risk Assessment, 13(1): 1-26, 1999.

[126] J.B. Smalley, B.S. Minsker, and D.E. Goldberg. Risked-based in situ bioremediation design using a noisy genetic algorithm. Water Resources Research, 36(10):3043-3051, 2000.

[127] T. Sperling, R. A. Freeze, J. Massmann, L. Smith, and B. James. Hydrogeological decision analysis: 3. application to design of a ground-water control system at an open pit mine. Ground Water, 30(3):376-389, 1992.

[128] C. I. Steefel and K. T. B. MacQuarrie. Approaches to modeling of reactive transport in porous media. In P. C. Lichtner, C. I. Steefel, and E. H. Oelkers, editors, Reactive Transport in Porous Media: General Principles and Applications to Geochemical Processes, pages 83-129. Mineralogical Society of America, Washington, DC, 1996.

[129] C. P. Stephens and W. Baritompa. Global optimization requires global information. J. Optim. Theory Appl., 96:575-588, 1998.

[130] R. A. Steuer. Multiple criteria optimization: theory, computation. Wiley, New York, 1986.

[131] D.E. Stoneking, G.L. Bilbro, R.J. Trew, P. Gilmore, and C. T. Kelley. 
Yield optimization using a GaAs process simulator coupled to a physical device model. IEEE Transactions on Microwave Theory and Techniques, 40:1353-1363, 1992.

[132] N.-Z. Sun and W. W. G. Yeh. A stochastic inverse solution for transient groundwater flow: Parameter identification and reliability analysis. Water Resources Research, 28(12):3269-3280, 1992.

[133] Y.-H. Sun, M. W. Davert, and W. W. G. Yeh. Soil vapor extraction system design by combinatorial optimization. Water Resources Research, 32(6):1863-1873, 1996.

[134] C. Tiedeman and S. M. Gorelick. Analysis of uncertainty in optimal groundwater contaminant capture design. Water Resources Research, 29(7):2139-2153, 1993.

[135] V. Torczon. Multidirectional Search. PhD thesis, Rice University, Houston, Texas, 1989.

[136] V. Torczon. On the convergence of the multidimensional direct search. SIAM J. Optim., 1:123-145, 1991.

[137] B. J. Wagner and S. M. Gorelick. Optimal groundwater quality management under parameter uncertainty. Water Resources Research, 23 (7):1162-1174, 1987.

[138] B. J. Wagner and S. M. Gorelick. Reliable aquifer remediation in the presence of spatially variable hydraulic conductivity: From data to design. Water Resources Research, 25(10):2211-2225, 1989.

[139] J. M. Wagner, U. Shamir, and H. R. Nemati. Groundwater quality management under uncertainty: Stochastic programming approaches and the value of information. Water Resources Research, 28(5):1233-1246, 1992.

[140] W. Wang and D. P. Ahlfeld. Optimal groundwater remediation with well location as a decision variable - model development. Water Resources Research, 30(5):1605-1618, 1994.

[141] R. Willis and W. W-G. Yeh. Groundwater Systems Planning and Management. Prentice-Hall, Englewood Cliffs, New Jersey, 1987.

[142] T. A. Winslow, R. J. Trew, P. Gilmore, and C. T. Kelley. Doping profiles for optimum class B performance of GaAs mesfet amplifiers. In Proceedings IEEE/Cornell Conference on Advanced Concepts in High Speed Devices and Circuits, pages 188-197. IEEE, 1991.

[143] Y. Y. Xiang, J. F. Sykes, and N. R. Thomson. Alternative formulations for optimal ground water remediation design. Journal of Water Resources Planning and Management - ASCE, 121(2):171-181, 1995.

[144] J.-H. Yoon and C. A. Shoemaker. Comparison of optimization methods for ground-water bioremediation. Journal of Water Resources Planning and Management, 125(1):54-63, 1999.

[145] D.M. Yu, M.and Rizzo and D.E. Dougherty. Multi-period objectives and groundwater remediation using samoa: tandem simulated annealing and extended cutting plane method for containment with cleanup. In XII International Conference on Computational Methods in Water Resources, pages 332-340. Elsevier, 1998. 
[146] C. Q. Zhen and J. G. Uber. Reliability of remediation designs in presence of modeling error. Journal of Water Resources Planning and Management - ASCE, 122(4):253-261, 1996.

[147] C. Zheng and P. P. Wang. An integrated global and local optimization approach for remediation system design. Water Resources Research, 35 (1):137-148, 1999. 